\title{
THE CHOW GROUP OF THE MODULI SPACE OF MARKED CUBIC SURFACES
}

\author{
ELISABETTA COLOMBO AND BERT VAN GEEMEN
}

\begin{abstract}
Naruki gave an explicit construction of the moduli space of marked cubic surfaces, starting from a toric variety and proceeding with blow ups and contractions. Using his result, we compute the Chow groups and the Chern classes of this moduli space. As an application we relate a recent result of Freitag on the Hilbert polynomial of a certain ring of modular forms to the Riemann-Roch theorem for the moduli space.
\end{abstract}

Dedicated to the memory of our friend Fabio Bardelli

Following on the work of Allcock, Carlson and Toledo [ACT], which identified the moduli space of marked cubic surfaces $\mathcal{M}$ as a ball quotient, there has been a renewed interest in moduli spaces of cubic surfaces. In particular, Allcock and Freitag $[\mathrm{AF}]$ found a projective embedding of $\mathcal{M}$ using new results of Borcherds on modular forms. This map was actually described earlier by Coble, who identified $\mathcal{M}$ with the moduli space of six points in $\mathbf{P}^{2}$. We will thus call this map the CAF-map.

The moduli space $\mathcal{M}$ is smooth except for 40 singular points, the cusps. Blowing up the cusps, one obtains a smooth projective variety $\mathcal{C}$ which we refer to as Naruki's cross ratio variety. Using basic work of Cayley on cubic surfaces and associated projective invariants, certain cross ratios, Naruki $[\mathrm{N}]$ realized that $\mathcal{C}$ could be obtained from a toric variety associated to the root system $D_{4}$ via a process of explicit blow ups and contractions. Moreover he showed that there is a biregular action of $W\left(E_{6}\right)$ on $\mathcal{C}$. His description of $\mathcal{C}$ is at the basis of this paper.

We determine the Chow groups of $\mathcal{C}$ and the spaces of $W\left(E_{6}\right)$-invariant cycles in section 2. In section 3 we consider the exceptional divisors in $\mathcal{C}$ over the cusps in $\mathcal{M}$, which we call cusp divisors (these are actually very simple varieties, being the product of three $\mathbf{P}^{1}$ s). We study the 36 boundary divisors, which parametrize nodal cubic surfaces, in section 4 . The image of such a divisor in $\mathcal{M}$ is isomorphic to the Segre cubic in $\mathbf{P}^{4}$. We use the information obtained on these divisors to compute the Chern classes of $\mathcal{C}$ in section 5 . In section 6.3 we consider the 45 tritangent divisors, these parametrize cubics with an Eckardt point (a point on the cubic surface through which 3 lines pass). All these divisors correspond, in a $W\left(E_{6}\right)$-equivariant way, to points in a finite projective geometry. For the convenience of the reader we added some tables at the end of the paper which give such a correspondence.

The main application of our results is an explicit form of the Riemann-Roch theorem on the fourfold $\mathcal{C}$ in section 5 . It allows us to compute the Euler characteristic of integral multiples of the divisor class which gives the CAF-map. The quartic polynomial we found (see Theorem 6.2) agrees with the Hilbert function of a certain graded ring of modular forms associated to 
the CAF-map which was recently determined by Freitag $[\mathrm{Fr}]$. We also obtain some results on the Picard group of the moduli space of smooth marked cubic surfaces in section 2.5.

Finally, we should probably point out that in using Naruki's description, we do not (need to) consider families of cubic surfaces and the 'tautological' classes associated to them. This is somewhat unfortunate, as this might give more insight in the structure of the Chow groups of $\mathcal{C}$ viewed as compactification of a moduli space.

\section{The CROSS RATIO VARIETY}

1.1. The toric variety $\tilde{T}$. The starting point of Naruki's construction is the smooth toric variety $T \cong\left(\mathbf{C}^{*}\right)^{4} \hookrightarrow \tilde{T}$ defined by the fan in the weight lattice $N \cong \mathbf{Z}^{4}$ of the root system of type $D_{4}$ whose 4-dimensional cones are the 192 Weyl chambers, see $[\mathrm{N}]$ and [vG], section 2. This fan has 48 edges (i.e. 1-dimensional cones), which correspond to the divisors in the boundary of $\tilde{T}$. Identifying $N \otimes_{\mathbf{z}} \mathbf{R}$ with $\mathbf{R}^{4}$, with standard basis $\epsilon_{1}, \ldots, \epsilon_{4}$ and standard scalar product, let

$$
S:=\left\{ \pm \epsilon_{i}\right\} \cup\left\{\left( \pm \epsilon_{1} \pm \epsilon_{2} \pm \epsilon_{3} \pm \epsilon_{4}\right) / 2\right\}, \quad R:=\left\{ \pm \epsilon_{i} \pm \epsilon_{j}\right\}
$$

then $S$ and $R$ each have 24 elements and the half-lines they generate are the edges of the fan. The elements from $S \cup R$ generate the $\mathbf{Z}$-module $N$, for $\tau \in S \cup R$ we denote by $V(\tau)(\subset \tilde{T})$ the corresponding divisor.

The character group of $T$ is identified with $M:=\operatorname{Hom}(N, \mathbf{Z})$, the root lattice of $D_{4}$. A Z-basis of $M$ is

$$
e_{1}-e_{2}, \quad e_{2}-e_{3}, \quad e_{3}-e_{4}, \quad e_{3}+e_{4},
$$

where the $e_{i}$ are the dual basis of the $\epsilon_{i}$, the corresponding characters $T \rightarrow \mathbf{C}^{*}$ are usually denoted by $\lambda, \rho, \nu$ and $\mu$ respectively.

1.2. The cross ratio variety $\mathcal{C}$. Naruki's (smooth, projective) cross ratio variety $\mathcal{C}$ is obtained from the toric variety $\tilde{T}$ as follows $([\mathrm{N}], \S 10-12)$ :

$$
\mathcal{C} \stackrel{r}{\longleftarrow} \hat{T} \stackrel{\pi^{\prime \prime}}{\longrightarrow} \tilde{T}^{\prime \prime} \stackrel{\pi^{\prime}}{\longrightarrow} \tilde{T}^{\prime} \stackrel{\pi_{e}}{\longrightarrow} \tilde{T}
$$

The map $\pi_{e}$ is the blow up of $\tilde{T}$ in the identity element $e \in T$. The exceptional divisor $\pi_{e}^{-1}(e) \cong \mathbf{P}^{3}$ is denoted by $\mathbf{P}_{\mathrm{w}}^{3}$. The image in $\mathcal{C}$ of its strict transform $\hat{E}$ in $\hat{T}$ is a tritangent divisor denoted by $D_{\mathrm{w}}=D_{(16)}$.

The map $\pi^{\prime}$ is the blow up of $\tilde{T}^{\prime}$ in 12 smooth, disjoint, rational curves $C_{1}^{\prime}, \ldots, C_{12}^{\prime}$. We write $C_{j}^{\prime \prime}:=\left(\pi^{\prime}\right)^{-1}\left(C_{j}^{\prime}\right)$ for the exceptional divisor, the map $C_{j}^{\prime \prime} \rightarrow C_{j}^{\prime}$ induced by $\pi^{\prime}$ is a $\mathbf{P}^{2}$-bundle.

The map $\pi^{\prime \prime}$ is the blow up in 16 smooth disjoint surfaces $S_{1}^{\prime \prime}, \ldots, S_{16}^{\prime \prime} \subset \tilde{T}^{\prime \prime}$. Each exceptional divisor $C_{j}^{\prime \prime}$ meets 4 of the surfaces $S_{i}^{\prime \prime}$ in 4 disjoint rational curves. These 4 curves are sections of the $\mathbf{P}^{2}$-bundle $C_{j}^{\prime \prime} \rightarrow C_{j}^{\prime}$ which meet each fiber of the $\mathbf{P}^{2}$-bundle $C_{j}^{\prime \prime} \rightarrow C_{j}^{\prime}$ in 4 general points (cf. [N], Prop. 9.1, §10). The strict transforms $\hat{C}_{j}$ in $\hat{T}$ of the $C_{j}^{\prime \prime}$ are trivial bundles:

$$
\hat{C}_{j} \cong \mathbf{P}^{1} \times V \stackrel{\pi^{\prime} \circ \pi^{\prime \prime}}{\longrightarrow} \mathbf{P}^{1} \cong C_{j}^{\prime}
$$

here $V$ is the blow up of $\mathbf{P}^{2}$ in 4 points, the map induced by $\pi^{\prime} \circ \pi^{\prime \prime}$ is the projection on the first factor. The map $r: \hat{T} \rightarrow \mathcal{C}$ induces the projection $\hat{C}_{j} \rightarrow V$ on the second factor and $r$ is an isomorphism on the complement of the $12 \hat{C}_{i}^{\prime}$ 's ([N], p. 22, 23 and Prop. 11.3). 
The 16 exceptional divisors $\hat{S}_{i}$ of $\pi^{\prime \prime}$ in $\hat{T}$ map under $r$ to divisors in $\mathcal{C}$. The $W\left(E_{6}\right)$-orbit of these 16 divisors in $\mathcal{C}$ consists of 40 divisors, the other 24 are the images under $r$ of the strict transforms of the $V(\tau)$ 's with $\tau \in R([\mathrm{~N}]$, Prop. 11.2). We call these 40 divisors the cusp divisors of $\mathcal{C}$.

1.3. The marked moduli space $\mathcal{M}$. There is a morphism

$$
c: \mathcal{C} \longrightarrow \mathcal{M}
$$

where $\mathcal{M}$ is the moduli space of semistable marked cubic surfaces, which contracts the 40 cusp divisors to (singular) points (cf. [N], Introduction and $\S 12$ ), the cusps of $\mathcal{M}$. The map $c$ is an isomorphism between the complement of the cusp divisors in $\mathcal{C}$ and the complement of the 40 cusps of $\mathcal{M}$. The Weyl group $W\left(E_{6}\right)$ acts biregularly on both $\mathcal{C}$ and $\mathcal{M}$, the morphism $c$ is $W\left(E_{6}\right)$-equivariant.

There is a $W\left(E_{6}\right)$-equivariant embedding, the CAF-map

$$
F: \mathcal{M} \longrightarrow \mathbf{P}^{9}
$$

which was studied extensively by Coble, Allcock and Freitag [AF], [Fr2] and also in [vG].

1.4. The cusp divisors. The cross ratio variety $\mathcal{C}$ has 40 cusp divisors of $\mathcal{C}$, each of these is isomorphic to $\mathbf{P}^{1} \times \mathbf{P}^{1} \times \mathbf{P}^{1}$, cf. [N], $\S 12$.

1.5. The boundary divisors. A boundary divisor is an irreducible component of the closure of the set of marked cubics with a node. There are 36 such divisors in $\mathcal{C}, 24$ of which are obtained from the $V(\tau)$ with $\tau \in S$, the other 12 come from the subtori defined by $\alpha=1$ in $T$ where $\alpha$ is a positive root of $D_{4}$.

1.6. The tritangent divisors. A tritangent divisor is an irreducible component of the closure of the set of marked cubics with an Eckardt point, that is a point on a cubic surface where three lines meet. There are 45 such divisors in $\mathcal{C}$. One of them is obtained from $\mathbf{P}_{\mathrm{w}}^{3}$, the other 44 are defined by the explicit equations in [N], Table 3.

1.7. Incidence between divisors. The cusp, boundary and tritangent divisors can be very conveniently parametrized by points in a finite projective orthogonal geometry. Let

$$
b: \mathbf{F}_{3}^{5} \times \mathbf{F}_{3}^{5} \longrightarrow \mathbf{F}_{3}, \quad b(x, y)=\sum_{i} x_{i} y_{i}
$$

be the standard bilinear form on $\mathbf{F}_{3}^{5}$ and let $q(x)=b(x, x)$ be the associated quadratic form. The orthogonal group $O\left(\mathbf{F}_{3}^{5}, q\right)$ is isomorphic to $W\left(E_{6}\right) \times\{ \pm 1\}$. Note that in $[\mathrm{AF}]$ the quadratic form $x_{1}^{2}-x_{2}^{2}-\ldots-x_{5}^{2}$ is used, substituting $x_{2}:=x_{2}+x_{3}, x_{3}=x_{2}-x_{3}$ and similarly for $x_{4}, x_{5}$, one obtains $q(x)$ since $-2 \equiv 1 \bmod 3$.

As the nonzero elements in $\mathbf{F}_{3}$ are \pm 1 , the subsets

$$
N_{i}:=\left\{z \in \mathbf{P}\left(\mathbf{F}_{3}^{5}\right): q(z)=i\right\}
$$

for $i \in \mathbf{F}_{3}$ are well defined. It is easy to check that

$$
\sharp N_{0}=40, \quad \sharp N_{1}=45, \quad \sharp N_{-1}=36 .
$$

The group $W\left(E_{6}\right)$ acts transitively on each of these sets.

There is a natural $W\left(E_{6}\right)$-equivariant identification between $N_{0}$ and the set of cusps, $N_{1}$ and the set of tritangent divisors, $N_{-1}$ and the set of boundary divisors, see [AF], section 3 . 
There it is also shown that a cusp divisor and a boundary (tritangent) divisor have non-empty intersection iff the corresponding lines in $\mathbf{F}_{3}^{5}$ are perpendicular. In particular, a boundary divisor meets 10 and a tritangent divisor meets 16 cusp divisors.

Lemma 3.1 of $[\mathrm{AF}]$ shows that if $D_{z}$ and $D_{w}$ are boundary divisors corresponding to $z, w \in$ $N_{-1}$ then $D_{z} \cap D_{w}$ is non-empty iff the lines $z, w \subset \mathbf{F}_{3}^{5}$ are perpendicular. This implies that a boundary divisor meets 15 other boundary divisors.

A tritangent divisor has non-empty intersection with any other tritangent divisor ([vG], 6.7) and with any boundary divisor (for this it is most convenient to use the description of these divisors as images of long and short mirrors in the 4-ball from [AF], section 3 ).

If $v \in \mathbf{F}_{3}^{5}$ is a vector with $q(v) \neq 0$, the map $s_{v}: x \mapsto x+q(v) b(x, v) v$ is in $O\left(\mathbf{F}_{3}^{5}, q\right)$, note that $s_{v}=s_{-v}$. For $v \in N_{-1}$ the map $s_{v}$ corresponds to the reflection in a roots of $E_{6}$. For $v \in N_{1}$, the map $-s_{v}$ corresponds to an involution in $W\left(E_{6}\right)$. The divisor $D_{v}$, for $v \in N_{-1} \cup N_{1}$, is the unique divisor in the fixed point set of the involution $\pm s_{v}$ in $\mathcal{C}$.

\section{The Chow Groups}

2.1. For a smooth variety $X$ we denote by $A^{i}(X)$ the Chow group of codimension $i$-cycles modulo rational equivalence. For a ring $R$ we write $A^{i}(X)_{R}:=A^{i}(X) \otimes_{\mathbf{z}} R$. We determine the Chow groups of $\mathcal{C}$ in Theorem 2.4 , some results on $A^{2}(\mathcal{C})$ are actually proved in 4.16 . Various intersection numbers are computed in 4.13 and 3.2 .

2.2. There are three divisors on $\mathcal{C}$ which are obviously invariant under $W\left(E_{6}\right)$, they are:

$$
\hat{B}, \quad \hat{C}, \quad \hat{T}
$$

which are the sum of the 36 boundary divisors, the sum of the 40 cusp divisors and the sum of the 45 tritangent divisors (actually we already used the notation $\hat{T}$ in 1.2 , however no confusion is possible). Theorem 2.4.2 shows that these classes are linearly dependent in $A^{1}(\mathcal{C})$.

2.3. Symmetrizing. Let $D$ be a divisor on $\mathcal{C}$ which is a linear combination of tritangent, boundary and cusp divisors. If the class of $D$ in the Chow group $A^{1}(\mathcal{C})$ is invariant, then $\sigma^{*} D$ has the same class for any $\sigma \in W\left(E_{6}\right)$. Therefore the class of $D$ is the same as the class of the sum over $\sigma \in W\left(E_{6}\right)$ of the $\sigma^{*} D$ divided by $\sharp W\left(E_{6}\right)$.

Since $W\left(E_{6}\right)$ permutes the 45 tritangent divisors transitively (and similarly the 36 boundary divisors as well as the 40 cusp divisors) the net result is that in $D$ we replace each tritangent divisor by $\frac{1}{45} \hat{T}$ etc.

\subsection{Theorem.}

(1) The Chow groups of the cross ratio variety $\mathcal{C}$ are free $\mathbf{Z}$-modules of rank:

$$
\operatorname{rk} A^{0}(\mathcal{C})=\operatorname{rk} A^{4}(\mathcal{C})=1, \quad \operatorname{rk} A^{1}(\mathcal{C})=\operatorname{rk} A^{3}(\mathcal{C})=61, \quad \operatorname{rk} A^{2}(\mathcal{C})=147
$$

(2) The subgroups of $W\left(E_{6}\right)$-invariant classes have rank:

$$
\operatorname{rk} A^{i}(\mathcal{C})^{W\left(E_{6}\right)}=1, \quad \operatorname{rk} A^{j}(\mathcal{C})^{W\left(E_{6}\right)}=2 \quad(i=0,4, \quad j=1,2,3) .
$$

The classes $\hat{B}^{i}, \hat{C}^{i}$ are a basis of $A^{i}(\mathcal{C})_{\mathbf{Q}}^{W\left(E_{6}\right)}$, we have:

$$
\hat{T}=(25 \hat{B}+27 \hat{C}) / 4 \in A^{1}(\mathcal{C}), \quad \hat{B} \hat{C}=-3 \hat{C}^{2} \in A^{2}(\mathcal{C}) .
$$


(3) The canonical class of the cross ratio variety is:

$$
K_{\mathcal{C}}=(-\hat{B}+\hat{C}) / 4 .
$$

(4) The decomposition of $A^{1}(\mathcal{C})$ and $A^{3}(\mathcal{C})$ into irreducible $W\left(E_{6}\right)$-representations is:

$$
A^{1}(\mathcal{C})_{\mathbf{C}} \cong A^{3}(\mathcal{C})_{\mathbf{C}}=\mathbf{1} \oplus \mathbf{1} \oplus \mathbf{1} \mathbf{5}_{\mathbf{q}} \oplus \mathbf{2 0} \mathbf{p} \oplus \mathbf{2} \mathbf{4}_{\mathbf{p}}
$$

(5) The cusp divisors are linearly independent in $A^{1}(\mathcal{C})$, the 40-dimensional subspace of $A^{1}\left(\mathcal{C}_{\mathbf{C}}\right)$ which they span decomposes as:

$$
1 \oplus 15_{\mathbf{q}} \oplus \mathbf{2 4 _ { \mathbf { p } }} .
$$

Similarly, the boundary divisors span a 36-dimensional subspace of $A^{1}(\mathcal{C})_{\mathbf{C}}$ which decomposes as:

$$
1 \oplus \mathbf{1 5} \mathbf{q}_{\mathbf{q}} \oplus \mathbf{2 0} \mathbf{p}
$$

and the tritangent divisors span a 45-dimensional subspace:

$$
1 \oplus 20_{\mathbf{p}} \oplus \mathbf{2 4 _ { \mathbf { p } }}
$$

Proof. The construction of $\mathcal{C}$ via the diagram in 1.2 shows that the Chow groups of $\mathcal{C}$ are free $\mathbf{Z}$-modules of finite rank and that $A^{i}(\mathcal{C}) \cong H^{2 i}(\mathcal{C}, \mathbf{Z})$ whereas $H^{2 i+1}(\mathcal{C}, \mathbf{Z})=0$. Since all varieties in the diagram in 1.2 , are smooth, so $A^{1} \cong A^{3}$, we concentrate on $A^{1}$ and $A^{2}$.

The rank of $A^{1}(\tilde{T})$ is given by the number of edges in the fan defining $\tilde{T}$, which is 48 , minus the rank of the torus, which is 4 . The Euler characteristic of $\tilde{T}$ is equal to the number of maximal cones ([Fu2], p.59), which is 192. Hence the rank of $A^{2}$ is $192-2-2 \cdot 44$ :

$$
\operatorname{rk} A^{1}(\tilde{T})=44, \quad \operatorname{rk} A^{2}(\tilde{T})=102 .
$$

The Chow groups of the blow up $\tilde{T}^{\prime}$ of $\tilde{T}$ in the identity element are $A^{i}\left(\tilde{T}^{\prime}\right) \cong A^{i}(\tilde{T}) \oplus A^{i}\left(\mathbf{P}_{\mathrm{w}}^{3}\right)$, for $i=1,2$ hence the ranks increase by 1 .

The variety $\tilde{T}^{\prime \prime}$ is obtained from $\tilde{T}^{\prime}$ by blowing up the 12 disjoint $C_{j}^{\prime}$ 's, each of which is a $\mathbf{P}^{1}$, hence we get:

$$
\operatorname{rk} A^{1}\left(\tilde{T}^{\prime \prime}\right)=45+12=57, \quad \operatorname{rk} A^{2}\left(\tilde{T}^{\prime \prime}\right)=103+24=127 .
$$

Next one blows up 16 surfaces $S_{i}^{\prime \prime}$ in $\tilde{T}^{\prime \prime}$ to obtain the variety $\hat{T}$. These surfaces are the strict transforms in $\tilde{T}^{\prime \prime}$ of surfaces $S_{i} \subset \tilde{T}$. Each $S_{i}$ is the closure of a subtorus (for example $\nu=\rho=1$ defines an $S_{i}$, the first table in 7.6 lists all 16 surfaces). Using the description of the fan defining $\tilde{T}$ one finds that $\operatorname{rk} A^{1}\left(S_{i}\right)=4$ for all $i$. These surfaces all contain $e \in \tilde{T}$ hence $\operatorname{rk} A^{1}\left(S_{i}^{\prime}\right)=5$, where $S_{i}^{\prime}$ is the strict transform of $S_{i}$ in $\tilde{T}^{\prime}$. Each $C_{j}^{\prime}$ is the strict transform of the closure $C_{j}$ of a 1-dimensional subtorus of $\tilde{T}([\mathrm{~N}], \mathrm{p} .20)$, hence $C_{j}$ is either contained in $S_{i}$ or meets it in $e$. As $e$ gets blown up, an $S_{i}^{\prime}$ either contains a $C_{j}^{\prime}$ or they are disjoint. Thus $\pi^{\prime \prime}: S_{i}^{\prime \prime} \rightarrow S_{i}^{\prime}$ is an isomorphism and $\operatorname{rk} A^{1}\left(S_{i}^{\prime \prime}\right)=5$. Therefore:

$$
\operatorname{rk} A^{1}(\hat{T})=57+16=73, \quad \operatorname{rk} A^{2}(\hat{T})=127+5 \cdot 16=207 .
$$

Finally we consider the blow down $r: \hat{T} \rightarrow \mathcal{C}$, it contracts the $12 \hat{C}_{j} \cong \mathbf{P}^{1} \times V$ in $\hat{T}$ to the surfaces $V$ in $\mathcal{C}$. Since $V$ is a $\mathbf{P}^{2}$ blown up in 4 points we get:

$$
\operatorname{rk} A^{1}(\mathcal{C})=73-12=61, \quad \operatorname{rk} A^{2}(\mathcal{C})=207-5 \cdot 12=147
$$


A relation between the invariant divisor classes can be obtained from the divisor of the rational function $\lambda-1$ on $\mathcal{C}$, see section 7.2. This divisor can be written as $(\lambda-1)_{0}-(\lambda-1)_{\infty}$ where $(\lambda-1)_{0}$ is the sum of one tritangent, one boundary and 4 cusp divisors, whereas $(\lambda-1)_{\infty}$ is the sum of 6 boundary and 9 cups divisors, one of which has multiplicity two. Symmetrizing this relation in $A^{1}(\mathcal{C})$, we get:

$$
0=\frac{1}{45} \hat{T}+\frac{1}{36}(1-6) \hat{B}+\frac{1}{40}(4-10) \hat{C},
$$

from which we get the relation $4 \hat{T}=25 \hat{B}+27 \hat{C}$ stated in the theorem. The relation in $A^{2}$ will be proved in Proposition 3.2 by restriction to a cusp divisor.

The canonical divisor of the toric variety $\tilde{T}$ is minus the sum of the 48 divisors $V(\tau)([\mathrm{Fu} 2]$, $\S 4.3), \tau \in R \cup S$, half of which give boundary divisors, the others give cups divisors. Blowing up the origin, we must add $3 \mathbf{P}_{\mathrm{w}}^{3}$ to the strict transform of $K_{\tilde{T}}$ to obtain $K_{\tilde{T}^{\prime}}([\mathrm{GH}]$, Lemma in Chap. 1.4, p.187). The exceptional divisors for $\pi^{\prime}$ are contracted by $r$, so these do not contribute, however we must add the 16 cups divisors which come from the blow up of the surfaces in $\tilde{T}^{\prime \prime}$. Symmetrizing the result we get:

$$
K_{C}=\frac{3}{45} \hat{T}+\frac{-24}{36} \hat{B}+\frac{1}{40}(-24+16) \hat{C}=(-\hat{B}+\hat{C}) / 4
$$

where we used the relation obtained before to eliminate $\hat{T}$. A nice, explicit, anti-canonical divisor is given at the end of 7.1.

From the construction of $\mathcal{C}$ it is clear that $A^{1}(\mathcal{C})$ is generated by the tritangent, boundary and cusp divisors. The divisor of $(\lambda-1)$ on $\mathcal{C}$ contains exactly one tritangent divisor with multiplicity one and this shows that the 36 boundary divisors and the 40 cusp divisors suffice to generate $A^{1}(\mathcal{C})$. Since $\operatorname{rk} A^{1}(\mathcal{C})=61$, there must be 15 independent relations between the boundary and cusp divisors. One such relation, which involves both boundary and cusp divisors, is given by the divisor of the rational function $\lambda$ (see 7.1), others can be obtained by applying $W\left(E_{6}\right)$ to this relation.

The group $W\left(E_{6}\right)$ permutes the boundary and the cusp divisors, the decomposition of these permutation representations can be found in the atlas [At], p. [26], $U_{4}(2)$, see also [F] whose notation we use. The permutation representations on the 36 boundary components and the 45 tritangents are denoted by $\chi^{(36)}$ and $\chi^{(45)}$ in $[\mathrm{F}], \mathrm{p} .100$. The group $W\left(E_{6}\right)$ has two permutation representations of degree 40 (see $[\mathrm{At}]$ ), the one which corresponds to the 40 cusp divisors is not $\chi^{(40)}$ in $[\mathrm{F}]$ but it is the one listed in the theorem, as can be verified by computing a few traces.

The only 15-dimensional $W\left(E_{6}\right)$ representation which the two permutation representations have in common is the irreducible representation $\mathbf{1 5}$, hence this must be the representation on the relations. Therefore the representation on $A^{1}(\mathcal{C})$ is the sum of the two permutation representations modulo $\mathbf{1 5}_{\mathbf{q}}$. In particular $A^{1}(\mathcal{C})^{W\left(E_{6}\right)}$ has rank 2 and is generated (over $\mathbf{Q}$ ) by $\hat{B}$ and $\hat{C}$. Since the permutation representation on the tritangents does not contain $\mathbf{1} \mathbf{5}_{\mathbf{q}}$ and since the relation provided by $\lambda-1$ involves both boundary and cusp divisors, the subrepresentations $\mathbf{2 0}_{\mathbf{p}}$ (which is in common with the boundary divisors) and $\mathbf{2 4} \mathbf{p}$ (which is in common with the cusps) cannot map to zero in $A^{1}(\mathcal{C})$, and finally since $\hat{T} \in A^{1}(\mathcal{C})$ is non-zero as well, we conclude that the tritangents divisors are independent in $A^{1}(\mathcal{C})$.

The proof of $\operatorname{rk} A^{2}(\mathcal{C})^{W\left(E_{6}\right)}=2$ will be given in 4.16 . 
2.5. Remark. Let $\mathcal{M}^{0} \subset \mathcal{M}$ be the moduli space of smooth marked cubic surfaces, so $\mathcal{M}^{0} \cong$ $\mathcal{C}-\operatorname{Support}(\hat{B}+\hat{C})$, and it is also the complement in $\mathcal{M}$ of the 36 boundary divisors. As we observed in the proof of the theorem, the first Chow group of $\mathcal{C}$ is generated by the classes of the boundary and cusp divisors. In particular,

$$
A^{1}\left(\mathcal{M}^{0}\right)=\operatorname{Pic}\left(\mathcal{M}^{0}\right)=0 .
$$

(Note that relations $\hat{T}=(25 \hat{B}+27 \hat{C}) / 4$ and $K_{\mathcal{C}}=(-\hat{B}+\mathcal{C}) / 4$ only show that $4 \hat{T}$ and $4 K_{\mathcal{C}}$ restrict to zero on $\left.\mathcal{M}^{0}\right)$. Since finite cyclic subgroups of $\operatorname{Pic}\left(\mathcal{M}^{0}\right)$ correspond to finite cyclic unramified coverings of $\mathcal{M}^{0}$, we conclude that the maximal abelian quotient of $\pi_{1}\left(\mathcal{M}^{0}\right)$ is trivial. See $[\mathrm{L}]$ for interesting results on this fundamental group.

2.6. The hyperplane class. We determine $(F \circ c)^{*} \mathcal{O}(1)$ where (cf. section 1.3)

$$
\mathcal{C} \stackrel{c}{\longrightarrow} \mathcal{M} \stackrel{F}{\longrightarrow} \mathbf{P}^{9} \text {. }
$$

From the work of Allcock and Freitag $[\mathrm{AF}]$ we know that, modulo cusp divisors, it is given by a so called cross divisor, that is

$$
(F \circ c)^{*} \mathcal{O}(1) \equiv D_{\alpha}+D_{\beta}+D_{\gamma}+D_{\delta}+D_{t} \quad \text { (modulo cusp divisors), }
$$

where $\alpha, \ldots, \delta \in N_{-1}$ and $t \in N_{1}$ are pairwise perpendicular. In particular, $D_{\alpha}, \ldots, D_{\delta}$ are boundary divisors and $D_{t}$ is a tritangent divisor.

The divisor class $(F \circ c)^{*} \mathcal{O}(1) \in A^{1}(\mathcal{C})$ is $W\left(E_{6}\right)$-invariant (since $F \circ c$ is $W\left(E_{6}\right)$-equivariant), and its expression in terms of invariant classes is given in the following proposition.

2.7. Proposition. The $W\left(E_{6}\right)$-invariant hyperplane class of the CAF map is given by:

$$
(F \circ c)^{*} \mathcal{O}(1)=(\hat{B}+3 \hat{C}) / 4 \text {. }
$$

Proof. The contribution of the cusp divisors to $D_{\alpha}+D_{\beta}+D_{\gamma}+D_{\delta}+D_{t}$ is the sum of the 24 cusp divisors $D_{n}$, each with multiplicity one, where $n \in N_{0}$ is perpendicular to at least one of element of the set $\{\alpha, \beta, \gamma, \delta, t\}$. This can be seen from a local computation using the explicit expression of the coordinate functions of $(F \circ c)$ (cf. [vG], 5.5 and the proof of Theorem 5.7).

Symmetrizing w.r.t. $W\left(E_{6}\right)$, as explained in 2.3 , we obtain the result.

\section{Cusp Divisors.}

3.1. According to Naruki, [N], each cusp divisor is isomorphic to $\mathbf{P}^{1} \times \mathbf{P}^{1} \times \mathbf{P}^{1}$. In the following proposition we prove the relation between $W\left(E_{6}\right)$-invariant codimension two classes (already stated in Theorem 2.4) and we determine some intersection numbers involving cusp divisors.

3.2. Proposition. Let $C_{0} \cong\left(\mathbf{P}^{1}\right)^{3}$ be a cusp divisor, and let

$$
D_{1}=\{0\} \times\left(\mathbf{P}^{1}\right)^{2}, \quad D_{2}=\mathbf{P}^{1} \times\{0\} \times \mathbf{P}^{1}, \quad D_{3}=\left(\mathbf{P}^{1}\right)^{2} \times\{0\} \quad\left(\in A^{1}\left(C_{0}\right)\right) .
$$

There are nine boundary divisors which have non-empty intersection with $C_{0}$. The class of the intersection of each of these with $C_{0}$ is one of the $D_{i}$ and

$$
C_{0} \hat{B}=3\left(D_{1}+D_{2}+D_{3}\right) \text {. }
$$

Let $B_{i}$ be a boundary divisor such that $B_{i} C_{0}=D_{i}$. Then, for $i \neq j$ :

$$
C_{0} B_{1} B_{2} B_{3}=1, \quad C_{0} B_{i}^{2} B_{j}=0, \quad C_{0} B_{i}^{3}=0, \quad C_{0}^{2} B_{i} B_{j}=-1, \quad C_{0}^{2} B_{i}^{2}=0 .
$$


Moreover,

Finally we have:

$$
C_{0}^{2}=-C_{0}\left(B_{1}+B_{2}+B_{3}\right), \quad C_{0}^{3} B_{i}=2, \quad C_{0}^{4}=-6
$$

$$
\hat{B} \hat{C}=-3 \hat{C}^{2} \in A^{2}(\mathcal{C}), \quad \hat{C}^{4}=-240
$$

Proof. The toric subvariety $V\left(\epsilon_{1}+\epsilon_{3}\right)$ in $\tilde{T}$ is defined by the fan consisting of the images in $\mathbf{R}^{4} /\left\langle\epsilon_{1}+\epsilon_{3}\right\rangle$ of all the cones containing $\epsilon_{1}+\epsilon_{3}$. One finds that this fan has six edges and that $V\left(\epsilon_{1}+\epsilon_{3}\right) \cong\left(\mathbf{P}^{1}\right)^{3}$. The divisor $V\left(\epsilon_{1}+\epsilon_{3}\right)$ is birationally isomorphic to a cusp divisor $C_{0}$ in $\mathcal{C}$ via the maps in the diagram in 1.2 above. One checks that each of these maps is an isomorphism on this divisor, hence $C_{0}$ is isomorphic to $\left(\mathbf{P}^{1}\right)^{3}$.

The six edges of the fan defining $C_{0}=V\left(\epsilon_{1}+\epsilon_{3}\right)$ define the classes $D_{i}$ (each twice) in $A^{1}\left(C_{0}\right)$. On the other hand, the edges correspond to non-empty intersections $V\left(\epsilon_{1}+\epsilon_{3}\right) \cap V(\tau)$. One verifies that $\tau \in S$, hence the $V(\tau)$ define boundary divisors. In particular, each $V\left(\epsilon_{1}+\epsilon_{3}\right) \cap V(\tau)$ is a $D_{i}$. That there are nine boundary divisors meeting $C_{0}$ follows from 1.7. In the notation of 7.6, $V\left(\epsilon_{1}+\epsilon_{3}\right)$ is labelled as [24.35.16]. The nine roots in this set correspond to the nine boundary divisors meeting $C_{0}$. These nine roots are in three orthogonal $A_{2}$ 's, the three divisors corresponding to the roots from one $A_{2}$ intersect $C_{0}$ in the same class in $A^{1}\left(C_{0}\right)$ because the corresponding divisors do not meet on $\mathcal{C}$. In particular $C_{0} \hat{B}=3\left(D_{1}+D_{2}+D_{3}\right)$.

Now it follows that $C_{0} B_{1} B_{2} B_{3}=1$ because the $D_{i}$ intersect transversely in one point on $C_{0}$, for $C_{0}^{2} B_{i} B_{j}$ see below. Finally $C_{0} B_{i}^{2} B_{j}=C_{0} B_{i}^{3}=C_{0}^{2} B_{i}^{2}=0$ since $D_{i}^{2}=0$ in $\left(\mathbf{P}^{1}\right)^{3}$.

To find the selfintersection $C_{0}^{2}$, we restrict the divisor of the rational function $\lambda$ (cf. 7.1) to $C_{0}=V\left(\epsilon_{1}+\epsilon_{3}\right)=D_{v}$ with $v=(1,-1,0,-1,0) \in N_{0}$. Using 1.7 and the tables in the Appendix to eliminate empty intersections, we find: $C_{0}\left(C_{0}+B_{1}+B_{2}+B_{3}\right)=0$ where the $B_{i}$ are the $V(\tau)$ with $\tau=\epsilon_{1},\left(\epsilon_{1}-\epsilon_{2}+\epsilon_{3}+\epsilon_{4}\right) / 2,\left(\epsilon_{1}-\epsilon_{2}+\epsilon_{3}-\epsilon_{4}\right) / 2$, which correspond to the roots $h_{345}, h_{136}$ and $h_{246}$. These three roots are in three distinct $A_{2}$ 's in [24.35.16] so $C_{0}^{2}=-\left(D_{1}+D_{2}+D_{3}\right)$. Using this relation twice we get:

$$
C_{0}^{3} B_{i}=C_{0}^{2}\left(-B_{1}-B_{2}-B_{3}\right) B_{i}=C_{0}\left(-B_{1}-B_{2}-B_{3}\right)^{2} B_{i}=2 .
$$

Therefore $C_{0}^{4}=C_{0}^{2} \cdot C_{0}\left(-B_{1}-B_{2}-B_{3}\right)=-3 \cdot 2=-6$. We also get $C_{0}^{2} B_{i} B_{j}=-\left(D_{1}+D_{2}+\right.$ $\left.D_{3}\right) D_{i} D_{j}=-1$ if $i \neq j$.

Since $\hat{C}$ is the sum of 40 disjoint cusp divisors, it follows that $\hat{C}^{4}=40 \cdot-6=-240$. Finally we observe that $C_{0} \hat{B}=3\left(D_{1}+D_{2}+D_{3}\right)=-3 C_{0}^{2}$, hence, summing over the 40 cusps, we get $\hat{C} \hat{B}=-3 \hat{C}^{2}$.

3.3. Remark. Recall from Theorem 2.4 that $r k\left(A^{2}(\mathcal{C})\right)=147$. We identify 120 independent codimension two cycles.

Let $C_{0} \cong\left(\mathbf{P}^{1}\right)^{3}$ be a cusp divisor and let $D_{i}=C_{0} B_{i} \in C_{0}$ be the three divisors on $C_{0}$ as in Proposition 3.2. Then $D_{i} D_{j}=-1$, if $i \neq j$, and $D_{i}^{2}=0$ in $A^{4}(\mathcal{C})$. Therefore the $D_{i}$ are independent in $A^{2}(\mathcal{C})$. As the 40 cusps are disjoint we get $3 \cdot 40=120$ independent codimension two classes on $\mathcal{C}$.

\section{THE BOUNDARY DIVISORS}

4.1. In this section we study the boundary divisors and we compute some intersection numbers on $\mathcal{C}$ in Theorem 4.13. 
4.2. The Chow group of a boundary divisor. Let $B_{0}$ be a boundary divisor on $\mathcal{C}$. Recall that the symmetric group $S_{6}$ acts on $B_{0}$. The divisor $B_{0}$ meets 15 other boundary divisors, 10 cusp divisors and the 45 tritangent divisors. We denote the sum of these by

$$
\hat{B}_{b}, \quad \hat{C}_{b}, \quad \hat{T}_{b}
$$

Then in $A^{1}\left(B_{0}\right)$ we have:

$$
\hat{B}_{\mid B_{0}}=\hat{B}_{b}+B_{0}^{2}, \quad \hat{C}_{\mid B_{0}}=\hat{C}_{b}, \quad \hat{T}_{\mid B_{0}}=\hat{T}_{b} .
$$

4.3. Proposition. The Chow groups of a boundary divisor $B_{0}$ are:

$$
A^{1}\left(B_{0}\right) \cong A^{2}\left(B_{0}\right) \cong \mathbf{Z}^{16} \text {. }
$$

We also have:

$$
A^{1}\left(B_{0}\right)^{S_{6}} \cong A^{2}\left(B_{0}\right)^{S_{6}} \cong \mathbf{Z}^{2}
$$

$\hat{B}_{b}^{i}, \quad \hat{C}_{b}^{i}$ are a basis of $A^{i}\left(B_{0}\right)_{\mathbf{Q}}^{S_{6}}$ for $i=1,2$ and $\hat{B}_{b} \hat{C}_{b}=-3 \hat{C}_{b}^{2}$. The intersection numbers between invariant classes are:

$$
\begin{gathered}
\hat{B}_{b}^{3}=-165=-3 \cdot 5 \cdot 11, \quad \hat{B}_{b}^{2} \hat{C}_{b}=180=2^{2} \cdot 3^{2} \cdot 5, \\
\hat{B}_{b} \hat{C}_{b}^{2}=-60=-2^{2} \cdot 3 \cdot 5, \quad \hat{C}_{b}^{3}=20=2^{2} \cdot 5 .
\end{gathered}
$$

Moreover we have:

$$
c_{1}\left(N_{B_{0} / \mathcal{C}}\right)=B_{0}^{2}=-\left(\hat{B}_{b}+3 \hat{C}_{b}\right) / 5, \quad K_{B_{0}}=-\left(2 \hat{B}_{b}+\hat{C}_{b}\right) / 5 .
$$

Proof. To compute the Chow groups of a boundary divisor, we choose $B_{0}$ to be the strict transform of the divisor $V\left(\epsilon_{1}\right)$ on the toric variety $\tilde{T}$. The divisor $V\left(\epsilon_{1}\right)$ is the toric variety defined by the fan consisting of the images in $\mathbf{R}^{4} /<\epsilon_{1}>$ of all the cones which contain $\epsilon_{1}$. One finds that $V\left(\epsilon_{1}\right)$ is smooth and that the fan has 14 edges. Thus $\operatorname{rk} A^{1} V\left(\epsilon_{1}\right)=14-3=11$. This divisor does not contain $e \in \tilde{T}$, but it meets (transversally in one point) exactly one of the 12 curves which get blown up in $\tilde{T}^{\prime}$, it is $C_{1}^{\prime}: \mu=\nu=\rho=1$, hence $\operatorname{rk} A^{1}\left(V\left(\epsilon_{1}\right)^{\prime \prime}\right)=12$. The divisor $V\left(\epsilon_{1}\right)^{\prime \prime}$ in $\tilde{T}^{\prime \prime}$ meets exactly 4 of the 16 surfaces which get blown up, it meets of them in a smooth rational curve. Therefore $\mathrm{rk} A^{1} \widehat{V\left(\epsilon_{1}\right)}=12+4=16$. Since $\widehat{V\left(\epsilon_{1}\right)}$ intersects just one of the $\hat{C}_{i} \cong \mathbf{P}^{1} \times S$ (it is $\left.\hat{C}_{1}\right)$ in a copy of $V([\mathrm{~N}]$, p. 22$)$ and $r$ contracts $\hat{C}_{1}$ to $V$, we conclude that the blow down map $r: \hat{T} \rightarrow \mathcal{C}$ induces an isomorphism of smooth 3 -folds $\widehat{V\left(\epsilon_{1}\right)} \cong B_{0}$. Hence $A^{1}\left(B_{0}\right)$ and $A^{2}\left(B_{0}\right)$ have rank 16.

Note that $K_{V\left(\varepsilon_{1}\right)}$ is minus the sum of the divisors corresponding to the 14 rays of the fan (the strict transform of 8 of these divisors is a boundary divisor, the other 6 are cusp divisors). From the formula for the canonical divisor of a blow up we find that $K_{V\left(\epsilon_{1}\right)^{\prime \prime}}$ is obtained from the strict transform of $K_{V\left(\varepsilon_{1}\right)}$ by adding twice the class of the (irreducible) exceptional divisor, this exceptional divisor is a boundary divisor. Finally $K_{\widehat{V\left(\epsilon_{1}\right)}}=K_{B_{0}}$ is obtained by adding 4 cusp divisors to this. Since $K_{B_{0}}$ is $S_{6}$-invariant, we can symmetrize w.r.t. $S_{6}$ :

$$
K_{B_{0}}=\frac{1}{15}(-8+2) \widehat{B}_{b}+\frac{1}{10}(-6+4) \widehat{C}_{b}=-\left(2 \hat{B}_{b}+\hat{C}_{b}\right) / 5 \text {. }
$$

The selfintersection $B_{0}^{2}$ can be computed from the restriction of the divisor of $\lambda$ on $\mathcal{C}$ (cf. 7.1) to $B_{0}$. As $B_{0}=r_{*} \widehat{V\left(\epsilon_{1}\right)}$ occurs in the divisor of zeroes with multiplicity one, the relation $0=B_{0} \cdot(\lambda)$ implies that $B_{0}^{2}$ is a linear combination of 4 boundary divisors in $B_{0}$ with coefficient 
-1 and 1 boundary divisor with coefficient 1, as well as 4 cusp divisors in $B_{0}$ with coefficient -1 and 1 with coefficient -2 . Symmetrizing w.r.t. $S_{6}$ we get:

$$
B_{0}^{2}=-\frac{1}{15}(4-1) \widehat{B}_{b}-\frac{1}{10}(4+2) \widehat{C}_{b}=-\left(\hat{B}_{b}+3 \hat{C}_{b}\right) / 5 .
$$

It is amusing to check that these expressions for $K_{\mathcal{C}}, K_{B_{0}}$ and $B_{0}^{2}$ do indeed satisfy the adjunction formula:

$$
K_{B_{0}}=\left(K_{\mathcal{C}}+B_{0}\right)_{\mid B_{0}} .
$$

See 4.8 for the $S_{6}$-invariant classes and 4.11 for the intersection numbers.

4.4. The Segre cubic. The Segre cubic is the cubic threefold $S$ in $\mathbf{P}^{5}$ defined by the equations:

$$
S: \quad x_{1}+\ldots+x_{6}=x_{1}^{3}+\ldots+x_{6}^{3}=0 .
$$

It is well know that any boundary divisor is isomorphic to the blow up of $S$ in its 10 nodes (the points with three coordinates +1 and three coordinates -1 ). In fact, the image of $B_{0}$ in $\mathcal{M} \subset \mathbf{P}^{9}$ is the Segre cubic (cf. [vG], 6.2). The 10 nodes are the images of the intersection of $B_{0}$ with 10 cusp divisors, each such intersection is isomorphic to the exceptional divisor over a node, thus to $\mathbf{P}^{1} \times \mathbf{P}^{1}$. The Segre cubic is obviously invariant under permutations of the $x_{i}$, this action of $S_{6}$ is induced by the action of a subgroup in $W\left(E_{6}\right)$ consisting of all the elements which fix $B_{0}$.

4.5. Boundary surfaces in $S$. The intersection of $B_{0}$ with a boundary divisor $B_{1}$ is, if nonempty, isomorphic to $V$, a $\mathbf{P}^{2}$ blown up in 4 general points. Thus $V$ maps to subvariety isomorphic to $\mathbf{P}^{2}$ in $S$ and this subvariety contains 4 of the singular points.

The divisor $B_{1}$ is contained in the fixed point set of an involution in $W\left(E_{6}\right)$ (a reflection in a root of $\left.E_{6}\right)$ ) and thus $B_{0} \cap B_{1}$ is contained in the fixed point set of an involution in $S_{6}$. Considering the involutions in $S_{6}$, we find that only the products of three commuting transpositions have a fixed point locus isomorphic to $\mathbf{P}^{2}$ and this $\mathbf{P}^{2}$ is linearly embedded. For $\sigma=(i j)(k l)(m n) \in S_{6}$ we have the fixed point set is

$$
\mathbf{P}_{\sigma}^{2}=\left\{x_{i}+x_{j}=x_{k}+x_{l}=x_{m}+x_{n}=0\right\} \quad \subset S .
$$

4.6. Parametrizing $S$. It is well-known (cf. [H], Theorem 3.1.2) that the blow up $B_{0}$ of the Segre threefold in the 10 nodes can be obtained as follows:

$$
S \longleftarrow B_{0} \stackrel{\rho_{2}}{\longrightarrow} B_{5} \mathbf{P}^{3} \stackrel{\rho_{1}}{\longrightarrow} \mathbf{P}^{3}
$$

here $\rho_{1}$ is the blow up of $\mathbf{P}^{3}$ in 5 general points $p_{1}, \ldots, p_{5}$ and $\rho_{2}$ is the blow up of $B_{5} \mathbf{P}^{3}$ in the strict transforms of the 10 lines spanned by pairs of the points. Note that this implies that $\operatorname{rk} A^{1}\left(B_{0}\right)=1+5+10=16$, in agreement with Prop. 4.3. The rational map $\mathbf{P}^{3}-\rightarrow S$ is given by the linear system of quadrics which contain the 5 points. 
4.7. The Chow group of $B_{0}$ (bis). We denote the exceptional fibers of $\rho_{1}$ by $V_{1}^{\prime}, \ldots, V_{5}^{\prime}$ and their strict transforms in $B_{0}$ by $V_{1}, \ldots, V_{5}$. The divisor $V_{1}^{\prime} \cong \mathbf{P}^{2}$ intersects the strict transform in $B_{5} \mathbf{P}^{3}$ of the 4 lines $\left\langle p_{1}, p_{i}\right\rangle(i=2, \ldots, 5)$ in 4 points in general position. Thus each $V_{i}$ is isomorphic to $V$.

Let $V_{i j}^{\prime \prime}=\left\langle p_{k}, p_{l}, p_{m}\right\rangle$ be the plane in $\mathbf{P}^{3}$ spanned by the points with complementary indices, so $\{i, \ldots, m\}=\{1, \ldots, 5\}$. Its strict transform $V_{i j}^{\prime}$ in $B_{5} \mathbf{P}^{3}$ has divisor class $H^{\prime}-V_{k}^{\prime}-V_{l}^{\prime}-V_{m}^{\prime}$, here $H^{\prime}$ is the pull-back of $\mathcal{O}(1)$ on $\mathbf{P}^{3}$. Its strict transform $V_{i j}$ in $B_{0}$ has class

$$
V_{i j}=H-V_{k}-V_{l}-V_{m}-C_{k l}-C_{k m}-C_{l m},
$$

where $C_{i j}$ is the exceptional divisor in $B_{0}$ over the strict transform of the line $\left\langle p_{i}, p_{j}\right\rangle$ (note that $V_{i j}^{\prime \prime}$ contains three of the 10 lines) and $H$ is the pull-back of $\mathcal{O}(1)$ to $B_{0}$. The intersection point $V_{i j}^{\prime \prime}$ with the line $\left\langle p_{i}, p_{j}\right\rangle$ is blown up by $\rho_{2}$, so $V_{i j}$ is also isomorphic to $V$.

The divisors $H, V_{1}, \ldots V_{5}$, and the $10 C_{i j}$ are a $\mathbf{Z}$-basis of $A^{1}\left(B_{0}\right)$, so it is easy to verify that the $5 V_{i}$ 's and the $10 V_{i j}$ 's are independent. It will be convenient to define $V_{i 6}:=V_{i}$, so we can talk simply about the $15 V_{i j}$.

Using explicit formulas for the quadrics on the 5 points (cf. [H], proof of Thm. 3.2.1), one verifies that the $15 V_{i j}$ map to the $15 \mathbf{P}_{\sigma}$. Thus we conclude, for example, that in $A^{1}\left(B_{0}\right)$ we have:

$$
\hat{B}_{b}=\sum_{i j}^{6} V_{i j}=10 H-5 \hat{V}-3 \hat{C}_{b}, \quad \text { with } \quad \hat{V}:=\sum_{i=1}^{5} V_{i}, \quad \hat{C}_{b}=\sum_{k l}^{5} C_{k l} .
$$

4.8. Invariant classes in $A^{1}\left(B_{0}\right)$. The group $S_{6}$ permutes the $\mathbf{P}_{\sigma}$ and thus the $15 V_{i j}$ 's transitively. Hence there is only a one dimensional $S_{6}$-invariant subspace in the subspace (of codimension 1) of $A^{1}\left(B_{0}\right)_{\mathbf{Q}}$ which they span. Therefore $\operatorname{dim} A^{1}\left(B_{0}\right)_{\mathbf{Q}}^{S_{6}} \leq 2$. Since also $\hat{C}_{b}$ is invariant and does not lie in the span of the $V_{i j}$ 's, we have $\operatorname{dim} A^{1}\left(B_{0}\right)_{\mathbf{Q}}^{S_{6}}=2$ and $\hat{B}_{b}, \hat{C}_{b}$ are a basis of the invariant classes.

4.9. The Picard group of $V$. Each of the 15 boundary surfaces $V_{i j}$ of $B_{0}$ is isomorphic to $V$ and it will be convenient to restrict divisor classes on $B_{0}$ (and on $\mathcal{C}$ ) to a $V_{i j}$ in order to compute intersection numbers, Chern classes etc.

Since $V$ is isomorphic to the blow up of $\mathbf{P}^{2}$ in 4 points, we have

$$
A^{1}(V) \cong \mathbf{Z} l \oplus \mathbf{Z} e_{1} \ldots \oplus \mathbf{Z} e_{4}
$$

with intersection product $l^{2}=1, e_{i}^{2}=-1, l \cdot e_{i}=e_{i} \cdot e_{j}=0$ if $1 \leq i, j \leq 4$ and $i \neq j$. Moreover, with $c:=e_{1}+\ldots+e_{4}$ :

$$
c_{1}(V)=-K_{V}=3 l-c, \quad c_{2}(V)=7,
$$

the formula for $K_{V}$ is standard ([HAG], V.3.3), for $c_{2}$ one can use $\chi\left(\mathcal{O}_{V}\right)=1$ and the Noether formula $\left([\mathrm{HAG}]\right.$, Appendix A, 4.1.2) $\chi\left(\mathcal{O}_{V}\right)=\left(K^{2}+c_{2}\right) / 12$.

4.10. Lemma. Let $V_{16}\left(\subset B_{0}\right)$ be the strict transform of the exceptional divisor $V_{1}^{\prime}\left(\subset B_{5} \mathbf{P}^{3}\right)$ over $p_{1} \in \mathbf{P}^{3}$. The restriction map on divisors is given by:

$$
A^{1}\left(B_{0}\right) \longrightarrow A^{1}\left(V_{16}\right), \quad \hat{B}_{b \mid V_{16}}=5 l-3 c, \quad \hat{C}_{b \mid V_{16}}=c, \quad V_{16}^{2}=-l \quad\left(\in A^{1}\left(V_{16}\right)\right) .
$$

In particular, the restriction map is injective on $S_{6}$-invariants. 
Proof. Since $V_{16}$ maps to a point in $\mathbf{P}^{3}$, the class $H$ maps to zero in $A^{1}\left(V_{16}\right)$. Moreover, $V_{16}$ does not intersect the divisors $V_{i 6}(i=2, \ldots, 5)$ nor the 4 divisors $V_{1 k}$ with $k=2, \ldots, 5$ nor the divisors $C_{k l}$ with $k, l \neq 1$, thus also these map to zero. The intersection $V_{16}$ and one of the 6 divisors $V_{k l}, 2 \leq k, l \leq 5$, is transversal along a divisor with class $l-e_{i}-e_{j}$ where $\{i, j, k, l\}=\{2, \ldots, 5\}$. Obviously $C_{1 j}$ maps to $e_{j-1}, j=2, \ldots, 5$ (up to permutation of indices).

It remains to determine the image of $V_{16} \in A^{1}\left(B_{0}\right)$, we write $V_{16}^{2}$ for this class. Using $K_{B_{0}}=(-2 / 5) \hat{B}_{b}+(1 / 5) \hat{C}_{b}$, which restricts to $\left.(-2 / 5)\left(V_{16}^{2}+6 l-2 c\right)\right)+(1 / 5) c$, and the adjunction formula :

$$
K_{V_{16}}=K_{B_{0 \mid V_{16}}}+V_{16}^{2} \quad \text { we get } \quad-3 l+c=\frac{3}{5} V_{16}^{2}-\frac{12}{5} l+c,
$$

hence $V_{16}^{2}=-l$.

4.11. Intersection numbers on $B_{0}$. It is now easy to compute the intersection numbers. If $D_{1}$ and $D_{2}$ are $S_{6}$-invariant divisors on $B_{0}$, then $D_{1} D_{2} \hat{B}_{b}=15 D_{1} D_{2} V_{16}$ since $\hat{B}_{b}$ is the sum of 15 surfaces in the $S_{6}$-orbit of $V:=V_{16}$. Recall that we may compute the intersection product $D_{1} D_{2} V$ on $B_{0}$ simply as $D_{1 \mid V} \cdot D_{2 \mid V}$ on $V$, in fact:

$$
D_{1} \cdot D_{2} \cdot V=D_{1} \cdot D_{2} \cdot j_{*} 1_{V}=j_{*}\left(j^{*}\left(D_{1} \cdot D_{2}\right) \cdot 1_{V}\right)=j_{*}\left(j^{*} D_{1} \cdot j^{*} D_{2}\right) \quad \in A^{3}\left(B_{0}\right)
$$

were $j: V \hookrightarrow B_{0}$ is the inclusion of $V$ in $B_{0}$ and $1_{V} \in A^{0}(V)$ is the class of $V, 1_{V}=[V]$, and we used the projection formula and the fact that $j^{*}$ is a ring homomorphism. Thus we find:

$$
\hat{B}_{b}^{3}=15 \hat{B}_{b \mid V} \hat{B}_{b \mid V}=15(5 l-3 c)^{2}=15(25+9 \cdot(-4))=165,
$$

Similarly, we get $\hat{B}_{b}^{2} \hat{C}_{b}=15(5 l-3 c) c=180$ and $\hat{B}_{b} \hat{C}_{b}^{2}=15 c^{2}=60$. It remains to compute $\hat{C}_{b}^{3}$, as $\hat{C}_{b}$ is the sum of 10 disjoint divisors in one $S_{6}$-orbit, this is $10 C_{b}^{3}$ where $C_{b}$ is a cusp divisor on $B_{0}$. But $C_{b}^{3}=C_{0}^{3} B_{0}=2$ by Proposition 3.2 .

4.12. Intersection numbers on $\mathcal{C}$. Using the results on the restriction to subvarieties we can now determine some intersection numbers on the cross ratio variety as well as its canonical class.

4.13. Theorem. The intersection numbers between invariant classes on the cross ratio variety $\mathcal{C}$ are:

$$
\begin{gathered}
\hat{B}^{4}=-12528=-2^{4} 3^{3} 29, \quad \hat{B}^{3} \hat{C}=6480=2^{4} 3^{4} 5, \quad \hat{B}^{2} \hat{C}^{2}=-2^{4} 3^{3} 5, \\
\hat{B} \hat{C}^{3}=720=2^{4} 3^{2} 5, \quad \hat{C}^{4}=-240=-2^{4} 3 \cdot 5 .
\end{gathered}
$$

Moreover, for boundary divisors $B_{0}, \ldots, B_{3}$ which have a non-empty intersection (that is, the corresponding lines in $\mathbf{F}_{3}^{5}$ are perpendicular) we have:

$$
B_{0}^{4}=-3, \quad B_{0}^{3} B_{1}=1, \quad B_{0}^{2} B_{1}^{2}=1, \quad B_{0}^{2} B_{1} B_{2}=-1, \quad B_{0} B_{1} B_{2} B_{3}=1 .
$$

Proof. We follow the strategy of section 4.11, replacing the role of the surface $V$ with that of the boundary divisor $B_{0}$. Since $\hat{B}$ is the sum of the 36 divisors in the $W\left(E_{6}\right)$-orbit of $B_{0}$ we get, for $W\left(E_{6}\right)$-invariant divisors $D_{1}, D_{2}, D_{3}$ :

$$
D_{1} D_{2} D_{3} \hat{B}=36 D_{1} D_{2} D_{3} B_{0}=36\left(D_{1} D_{2} D_{3}\right)_{\mid B_{0}}=36\left(D_{1 \mid B_{0}}\right)\left(D_{2 \mid B_{0}}\right)\left(D_{3 \mid B_{0}}\right) .
$$


Using that $\hat{B}_{\mid B_{0}}=\hat{B}_{b}+B_{0}^{2}=\left(4 \hat{B}_{b}-3 \hat{C}_{b}\right) / 5$ (cf. 4.2 and Prop. 4.3 ) we obtain:

$$
\hat{B}^{4}=36 \hat{B}_{\mid B_{0}}^{3}=\frac{36}{125}\left(4 \hat{B}_{b}-3 \hat{C}_{b}\right)^{3}=\frac{36}{125}(64 \cdot(-165)-\ldots)=-2^{4} 3^{3} 29,
$$

similarly one finds:

$$
\hat{B}^{3} \hat{C}=\frac{36}{25}\left(4 \hat{B}_{b}-3 \hat{C}_{b}\right)^{2} \hat{C}_{b}=6480, \quad \hat{B}^{2} \hat{C}^{2}=\frac{36}{5}\left(4 \hat{B}_{b}-3 \hat{C}_{b}\right) \hat{C}_{b}^{2}=-2160,
$$

and $\hat{B} \hat{C}^{3}=36 \hat{C}_{b}^{3}=720$. For $\hat{C}^{4}$ see Proposition 3.2.

The intersection numbers of the boundary divisors are easily determined by restriction to $B_{0}$ or $B_{0} B_{1}=V_{i j}$ (for some $i, j$ ), the restriction map $A^{1}\left(B_{0}\right) \rightarrow A^{1}\left(V_{i j}\right)$ is given in 4.10. Hence:

$$
B_{0}^{4}=N_{B_{0} / \mathcal{C}}^{3}=-\frac{1}{125}\left(\hat{B}_{b}+3 \hat{C}_{b}\right)^{3}=-3, \quad B_{0}^{3} B_{1}=N_{B_{0} / \mathcal{C}}^{2} \cdot V_{i j}=\frac{1}{25}(5 l-3 c+3 c)^{2}=1 .
$$

Similarly one computes the other intersection numbers.

4.14. It is now easy to compute the degree of the moduli space of marked cubics embedded in $\mathbf{P}^{9}$ by the CAF-system. This computation was already done in [Fr]. Freitag used two skew $\mathbf{P}^{4}$ 's in $\mathbf{P}^{9}$ (eigenspaces of a subgroup of index two of $W\left(E_{6}\right)$ ) and showed, using explicit generators of the ideal of $\mathcal{M} \subset \mathbf{P}^{9}$ and computer algebra, that the projection from one to the other induces a morphism $\mathcal{M} \rightarrow \mathbf{P}^{4}$ of degree 27 .

4.15. Corollary. The degree of the image in $\mathbf{P}^{9}$ of the moduli space $\mathcal{M}$ under the CAF-map is 27 .

Proof. From Theorem 2.6 it follows that the degree is given by

$$
((\hat{B}+3 \hat{C}) / 4)^{4}=\frac{1}{256}\left(\hat{B}^{4}+12 \hat{B}^{3} \hat{C}+\ldots+81 \hat{C}^{4}\right)=27
$$

where we used the intersection numbers from Theorem 4.13.

4.16. The $W\left(E_{6}\right)$-ivariants in $A^{2}(\mathcal{C})$. We already observed in 3.3 that there are 120 independent codimension 2 classes (obtained as $B_{i} C_{j}$ ) supported on the cusp divisors. It is easy to see that $W\left(E_{6}\right)$ permutes these classes transitively, and hence the subspace of invariant classes has dimension 1.

Next we consider the orthogonal complement (w.r.t. the intersection form) in $A^{2}$, it has dimension $147-120=27$. Classes in the complement are given for example by the selfintersections $B_{i}^{2}$ of the 36 boundary divisors (indeed, $B_{i} C_{j} B_{k}^{2}=0$, for any indices, by Proposition 3.2). A computation shows that the rank of the intersection matrix $\left(B_{i}^{2} B_{j}^{2}\right)$ is 21 . The group $W\left(E_{6}\right)$ permutes these classes and since the permutation character is $\mathbf{1} \oplus \mathbf{1 5}_{\mathbf{q}} \oplus \mathbf{2 0}_{\mathbf{p}}$, we conclude that there is in $A^{2}(\mathcal{C})_{\mathbf{Q}}$ a 21-dimensional subspace, perpendicular to the 120 subspace, on which $W\left(E_{6}\right)$ acts via $\mathbf{1} \oplus \mathbf{2 0}_{\mathbf{p}}$. In particular we found another invariant.

To get our hands on the remaining 6-dimensional subspace, we considered the image of the 306-dimensional Q-vectorspace $W$ generated by the $270=36 \cdot 15 / 2$ (non-empty) intersections $B_{i} B_{j}$ of boundary divisors and the $36 B_{i}^{2}$. The group $W\left(E_{6}\right)$ acts on $W$ by permuting the basis vectors. Using the previous theorem (and a computer) we found that the matrix of intersection products has rank 147, hence these classes span $A^{2}(\mathcal{C})_{\mathbf{Q}}$ (the 270 classes $B_{i} B_{j}$ span a subspace of codimension 1). Next one computes the kernel of $W \rightarrow A^{2}(\mathcal{C})_{\mathbf{Q}}$ (which is the kernel of the intersection matrix). Its perpendicular w.r.t. to the standard innerproduct on $W$ is a $W\left(E_{6}\right)$-invariant subspace which maps isomorphically onto $A^{2}(\mathcal{C})_{\mathbf{Q}}$. Now one computes 
the 6 -dimensional subspace which is perpendicular to the $120+21=141$-dimensional subspace we already found and one verifies that it is the standard 6-dimensional representation $\mathbf{6}_{\mathbf{p}}$ of $W\left(E_{6}\right)$.

A class in this subspace, which corresponds to the root $h$, is the class $S_{h}=S_{h}^{+}-S_{h}^{-}$, where $S_{h}^{+}$is the sum of the $15 \cdot 4=60$ classes $B_{i j} \cdot B_{i j k}(1 \leq i \leq j \leq 6$ and $k \neq i, j)$ and $S_{h}^{-}$is the sum of the 60 classes $B_{i j} \cdot B_{k l m}(1 \leq i \leq j \leq 6$ and $\{k, l, m\} \cap\{i, j\}=\emptyset)$, here we wrote $B_{m}$ for the boundary divisor corresponding to the root $h_{m}$ (cf. 7.4).

In any case, we can now conclude that $\operatorname{rk} A^{2}(\mathcal{C})^{W\left(E_{6}\right)}=2$. As a byproduct, we found that the complement of the 120 classes supported on the cusps has the representation $\mathbf{1} \oplus \mathbf{2 0}_{\mathbf{p}} \oplus \mathbf{6}_{\mathbf{p}}$, which is the permutation representation of $W\left(E_{6}\right)$ on the 27 lines (!), cf. [F], (5.2). For completeness sake, the representation on the 120 -dimensional subspace is $\mathbf{1} \oplus \mathbf{1 5}_{\mathbf{q}} \oplus \mathbf{2 0}_{\mathbf{p}} \oplus \mathbf{2 \mathbf { 4 } _ { \mathbf { p } }} \oplus \mathbf{6} \mathbf{0}_{\mathbf{p}}$.

\section{The Riemann-Roch Theorem}

5.1. Riemann-Roch. The Riemann-Roch theorem for a line bundle $\mathcal{O}_{\mathcal{C}}(D)$ on the cross ratio variety $\mathcal{C}$ is:

$$
\chi\left(\mathcal{O}_{\mathcal{C}}(D)\right)=\operatorname{deg}\left(\left(1+D+\frac{1}{2} D^{2}+\frac{1}{6} D^{3}+\frac{1}{24} D^{4}\right) \cdot\left(1+\frac{1}{2} c_{1}+\frac{1}{12}\left(c_{1}^{2}+c_{2}\right)+\frac{1}{24} c_{1} c_{2}+t d(\mathcal{T})_{4}\right)\right)_{4}
$$

where $t d(\mathcal{T})_{4}=\frac{-1}{720}\left(c_{1}^{4}-4 c_{1}^{2} c_{2}-3 c_{2}^{2}-c_{1} c_{3}+c_{4}\right)$, ([HAG], Appendix A.4) and $\mathcal{T}$ is the tangent bundle of $\mathcal{C}$. In particular, if $D=0$ we find $1=\chi\left(\mathcal{O}_{\mathcal{C}}\right)=t d(\mathcal{T})_{4}$. Hence to use the RiemannRoch theorem we only need to compute $c_{1}$ and $c_{2}$ of $\mathcal{C}$, and we will only indicate how we computed $c_{3}$ and $c_{4}$.

5.2. Chern classes. The Chern classes $c_{1}$ and $c_{2}$ can be computed easily by restriction to a divisor. In general, if $Y$ is a smooth divisor on $X$, the exact sequence

$$
0 \longrightarrow \mathcal{T}_{Y} \longrightarrow \mathcal{T}_{X \mid Y} \longrightarrow \mathcal{N}_{Y / X} \longrightarrow 0
$$

gives the following relations in $A^{*}(Y)$ :

$$
c_{1}(X)_{\mid Y}=c_{1}(Y)+Y^{2}, \quad c_{2}(X)_{\mid Y}=c_{1}(Y) \cdot Y^{2}+c_{2}(Y), \quad c_{3}(X)_{\mid Y}=c_{2}(Y) \cdot Y^{2}+c_{3}(Y)
$$

where $Y^{2}=c_{1}\left(N_{Y / X}\right) \in A^{1}(Y)$, the first relation is equivalent to the adjunction formula.

5.3. Proposition. The Chern classes of a boundary divisor $B_{0}$ are:

$$
c_{1}\left(B_{0}\right)=\left(2 \hat{B}_{b}+\hat{C}_{b}\right) / 5, \quad c_{2}\left(B_{0}\right)=\left(4 \hat{B}_{b}^{2}-36 \hat{C}_{b}^{2}\right) / 25, \quad c_{3}\left(B_{0}\right)=34
$$

Proof. To compute $c_{2}\left(B_{0}\right)$ we consider the divisor $Q \cong\left(\mathbf{P}^{1}\right)^{2}$ which lies over a cusp in the Segre threefold. We can identify $Q$ with the divisor $D_{1}=B_{0} \cap C_{0}$ as in Proposition 3.2. Only six of the nine boundary divisors, besides $B_{0}$, which meet $C_{0}$ intersect $Q$, they intersect it in $3 D_{1} D_{2}+3 D_{1} D_{3}=3 \Delta$ where $\Delta$ is the diagonal of $\left(\mathbf{P}^{1}\right)^{2}$. Thus $\hat{B}_{b}$ restricts to $3 \Delta$ on $Q$. Since $\hat{C}_{b}$ is the sum of the 10 disjoint cusp divisors on $B_{0}$, one of which is $Q$, its restriction to $Q$ is $Q^{2}=N_{Q / B_{0}}$. Since $K_{Q}=-2 \Delta$ the adjunction formula gives

$$
-2 \Delta=K_{B_{0} \mid Q}+Q^{2}=\left(\left(-2 \hat{B}_{b}-\hat{C}_{b}\right) / 5\right)_{\mid Q}+Q^{2}=\left(-6 \Delta+4 Q^{2}\right) / 5
$$

hence $Q^{2}=-\Delta$. Furthermore, since $\hat{B}_{b}^{2} \hat{C}_{b}=10 \cdot 18, \hat{C}_{b}^{3}=20$ and $\hat{C}_{b}$ is the sum of 10 disjoint copies of $Q$, we get $\hat{B}_{b}^{2} Q=18, \hat{C}_{b}^{2} Q=2$. 
Now we write $c_{2}\left(B_{0}\right)=n \hat{B}_{b}^{2}+m \hat{C}_{b}^{2}$ and use the relation 5.2 for $Q \subset B_{0}$ :

$$
\left(n \hat{B}_{b}^{2}+m \hat{C}_{b}^{2}\right)_{\mid Q}=c_{1}(Q) Q^{2}+c_{2}(Q)=(2 \Delta)(-\Delta)+4=0,
$$

hence $18 n+2 m=0$. On any smooth threefold we have $c_{1} c_{2} / 24=\chi(\mathcal{O})$. Since $\chi(\mathcal{O})=1$ for $B_{0}$ this implies

$$
24=\frac{1}{5}\left(2 \hat{B}_{b}+\hat{C}_{b}\right)\left(n \hat{B}_{b}^{2}+m \hat{C}_{b}^{2}\right)=-30 n-20 m .
$$

From these equations one finds $n=4 / 25$ and $m=-36 / 25$.

Recall that $B_{0}$ is obtained as a blow up in two different ways. One is described in section 4.6, the other in the proof of Proposition 4.3. In [Fu1], Thm. 15.4 the Chern classes of the blow up of a smooth threefold $Y$ in a point $p$ or in a smooth curve $X$ are given. Applying these formulas allows one to compute, in two different ways, $c_{3}\left(B_{0}\right)$ and we found the same result, $c_{3}\left(B_{0}\right)=34$, in both ways. This method also allowed us to verify the formula for $c_{2}\left(B_{0}\right)$ determined above.

5.4. Theorem. The Chern classes of the cross ratio variety $\mathcal{C}$ are:

$$
c_{1}(\mathcal{C})=(\hat{B}-\hat{C}) / 4, \quad c_{2}(\mathcal{C})=\left(\hat{B}^{2}-9 \hat{C}^{2}\right) / 8, \quad c_{3}(\mathcal{C})=\frac{13}{3 \cdot 96} \hat{B}^{3}+\frac{181}{96} \hat{C}^{3},
$$

and $c_{4}(\mathcal{C})=271$.

Proof. Since $c_{1}(\mathcal{C})=-K_{\mathcal{C}}$, the first result follows from Theorem 2.4. The expression for $c_{2}$ follows from the formula for $c_{2}(X)_{\mid Y}$ in 5.2 applied to $B_{0} \subset \mathcal{C}$ and the previous proposition. Similarly, since the Chern class $c_{3}$ is invariant under $W\left(E_{6}\right)$ it can be written as $a \hat{B}^{3}+b \hat{C}^{3}$ for certain $a, b \in \mathbf{Q}$. Restricting to $B_{0}$ and to a cusp divisor $C_{0}$ and using $c_{3}\left(B_{0}\right)=34, c_{3}\left(C_{0}\right)=8$ (which follows from $C_{0} \cong\left(\mathbf{P}^{1}\right)^{3}$ ) we find two equations for $a$ and $b$ from which $c_{3}$ can be determined. The class $c_{4}$ can be computed from the other $c_{i}$ and the relation $t d(\mathcal{T})_{4}=1$.

\section{Applications}

6.1. The CAF-linear system. In $[\mathrm{Fr}]$, Freitag determined the Hilbert function of the graded ring $\mathbf{C}\left[Y_{0}, \ldots, Y_{9}\right] / \mathcal{I}$, where $\mathcal{I}$ is the homogeneous ideal defining $\mathcal{M} \subset \mathbf{P}^{9}$. The Hilbert function coincides with the map $n \mapsto \chi\left(\mathcal{O}_{\mathcal{C}}(n H)\right)$, see the theorem below. This suggests that that the multiplication maps $\operatorname{Sym}^{n} H^{0}(H) \rightarrow H^{0}(n H)$ are surjective and that $H^{i}(n H)=0$ for $i, n>0$.

6.2. Theorem. Let $H=(\hat{B}+3 \hat{C}) / 4$ be the pull-back of the hyperplane section of $\mathcal{C} \rightarrow \mathbf{P}^{9}$. Then we have:

$$
\chi\left(\mathcal{O}_{\mathcal{C}}(n H)\right)=\frac{9}{8} n^{4}+\frac{9}{4} n^{3}+\frac{27}{8} n^{2}+\frac{9}{4} n+1 .
$$

Proof. This follows by direct computation from the Riemann-Roch formula.

6.3. Tritangent divisors. We haven't used the tritangent divisors to obtain the results above. However, applying the same methods we can describe the Chow groups of a tritangent divisor. We will denote the tritangent divisor in $\mathcal{C}$ obtained from $\mathbf{P}_{\mathrm{w}}^{3}$ in $\tilde{T}^{\prime}$ by $T_{0}$. The strict transform of the hyperplane class in $\mathbf{P}_{\mathrm{w}}^{3}$ will be denoted by $H_{\mathrm{w}} \in A^{1}\left(T_{0}\right)$. There is an identification $\mathbf{P}_{\mathrm{w}}^{3} \cong \mathbf{P}\left(Q\left(F_{4}\right) \otimes_{\mathbf{z}} \mathbf{C}\right)$, where $Q\left(F_{4}\right)$ is the root lattice of the root system system of type $F_{4}$ such that the action of $W\left(F_{4}\right)$ on $Q\left(F_{4}\right)$ induces the biregular action of $W\left(F_{4}\right)\left(\subset W\left(E_{6}\right)\right)$ on $T_{0}$ (cf. [vG], 6.4, 6.5). The root system $F_{4}$ has 24 short roots, these are the elements of $S$, and 
24 long roots, the elements of $R$, these form a subroot system of type $D_{4}$ in $F_{4}$. The 12 points in $\mathbf{P}\left(Q\left(F_{4}\right) \otimes_{\mathbf{z}} \mathbf{C}\right)$ defined by the short roots of $F_{4}$ are blown up to divisors in $T_{0}$. The sum of these 12 divisors in $T_{0}$ will be denoted by $\hat{B}_{t, e}$.

6.4. Proposition. The Chow groups of a tritangent divisor $T_{0}$ are:

$$
A^{1}\left(T_{0}\right) \cong A^{2}\left(T_{0}\right) \cong \mathbf{Z}^{29}
$$

Moreover we have:

$$
A^{1}\left(T_{0}\right)^{W\left(F_{4}\right)} \cong A^{2}\left(T_{0}\right)^{W\left(F_{4}\right)} \cong \mathbf{Z}^{3}
$$

and the classes $H_{\mathrm{w}}, \hat{B}_{t}:=\hat{B}_{\mid T_{0}}$ and $\hat{C}_{t}:=\hat{C}_{\mid T_{0}}$ are a basis of $A^{1}\left(T_{0}\right)_{\mathbf{Q}}^{W\left(F_{4}\right)}$.

The chern classes of $T_{0}$ are:

$$
-c_{1}\left(T_{0}\right)=K_{T_{0}}=2 H_{\mathrm{w}}-\frac{1}{2} \hat{B}_{t}-\frac{1}{2} \hat{C}_{t}, \quad c_{2}\left(T_{0}\right)=6 H_{\mathrm{w}}^{2}-2 H_{\mathrm{w}} \hat{C}_{t}-\hat{C}_{t}^{2}, \quad c_{3}\left(T_{0}\right)=92 .
$$

The hyperplane class of the CAF-map restricts to

$$
3 H_{\mathrm{w}}-\hat{B}_{t, e} .
$$

Proof. The 12 curves $C_{i}^{\prime} \subset \tilde{T}^{\prime}$ which are blown up in $\pi^{\prime}$ intersect the projective space $\mathbf{P}_{\mathrm{w}}^{3}$ in 12 points, these are the points defined by $S$. This adds 12 to the rank of $A^{1}$. Next in $\tilde{T}^{\prime \prime}$ the strict transform of $\mathbf{P}_{\mathrm{w}}^{3}$ meets the $16 S_{i}^{\prime \prime \prime}$ s in 16 smooth rational curves (these curves are the strict transforms of 16 lines in $\mathbf{P}_{\mathrm{w}}^{3}$, each line contains three of the points, on each point there are 4 lines). Thus for the strict transform $\hat{E}$ of $\mathbf{P}_{\mathrm{w}}^{3}$ in $\hat{T}$ we get $\operatorname{rk} A^{1}(\hat{E})=1+12+16=29$.

The intersection of $\hat{E}$ and a $\hat{C}_{i} \cong \mathbf{P}^{1} \times V$ is a copy of $V$ (cf. [N], p. 22). Since the contraction $r: \hat{T} \rightarrow \mathcal{C}$ induces the projection $\mathbf{P}^{1} \times V \rightarrow V$, the map $r$ induces an isomorphism $\hat{E} \cong T_{0}$. Thus also $\operatorname{rk} A^{1}\left(T_{0}\right)=29$. The rank of $A^{2}$ follows since $T_{0}$ is easily seen to be smooth by construction. The invariant classes are also obvious.

A tritangent divisor has non-empty intersection with each boundary divisor. The 12 divisors in $\tilde{T}^{\prime}$ obtained from the subtori $\alpha=1$ in $T$, with $\alpha$ a root of $D_{4}$, intersect $\mathbf{P}_{\mathrm{w}}^{3}$ in linear subspaces defined by the same root of $F_{4}$ ([vG], proof of Thm. 6.5$)$. We denote by $\hat{B}_{t, i}$ the sum of the twelve divisors in $T_{0}$ obtained in this way. The other $36-12=2 \cdot 12$ boundary divisors in $\mathcal{C}$ are obtained from the $V(\tau)$ where $\tau \in S$, these are paired by $\tau \leftrightarrow-\tau$. The image $C_{j}$ in $\tilde{T}$ of each of the twelve curves $C_{j}^{\prime}$ in $\tilde{T}^{\prime}$ has non-empty intersection with exactly two such boundary divisors, the two are in a pair and each intersection is transversal in one point. We denote the pair defined by $C_{j}$ by $\pm \tau_{j}$. The divisors $\hat{T}$ and $\widehat{V\left( \pm \tau_{j}\right)}$ are still disjoint, but each meets the divisor $\hat{C}_{j} \cong C_{j} \times V$ in a copy of $V$. In the final blow down $r: \hat{T} \rightarrow \mathcal{C}, \hat{C}_{j}$ is contracted onto $V$, hence this surface is the intersection of any two of $T_{0}, B_{\tau_{j}}$, and $B_{-\tau_{j}}$ where $B_{\tau}$ is the boundary divisor defined by $V(\tau)$. We denote the sum of these 12 surfaces by $\hat{B}_{t, e}$, then

$$
\hat{B}_{t}=\hat{B}_{t, i}+2 \hat{B}_{t, e} .
$$

Using the divisor of $\lambda-1$ (cf. 7.1) restricted to $T_{0}$ we find that $T_{0}^{2}$ is is the sum of $(-1)$ times one of the twelve divisors in $\hat{B}_{t, i}$ (it is $\left.D_{\lambda}^{1} \cap T_{0}\right)$ ), minus the sum of 4 divisors in $\hat{C}_{t}$ and plus 
the sum of 6 divisors from $\hat{B}_{t, e}$. Symmetrizing w.r.t. $W\left(F_{4}\right)$ (so we replace each divisor in $\hat{B}_{t, i}$ by $(1 / 12) \hat{B}_{t, i}$ etc. $)$ we find:

$$
T_{0}^{2}=\frac{-1}{12} \hat{B}_{t, i}+\frac{6}{12} \hat{B}_{t, e}-\frac{4}{16} \hat{C}_{t}=\frac{-1}{12} \hat{B}_{t, i}+\frac{1}{2} \hat{B}_{t, e}-\frac{1}{4} \hat{C}_{t}
$$

The canonical divisor of $T_{0}$ can be obtained from the adjunction formula for $T_{0} \subset \mathcal{C}$ :

$$
K_{T_{0}}=K_{\mathcal{C} \mid T_{0}}+T_{0}^{2}=\frac{1}{4}\left(-\hat{B}_{t, i}-2 \hat{B}_{t, e}+\hat{C}_{t}\right)-\frac{1}{12} \hat{B}_{t, i}+\frac{1}{2} \hat{B}_{t, e}-\frac{1}{4} \hat{C}_{t}=\frac{-1}{3} \hat{B}_{t, i} .
$$

The canonical divisor of $T_{0}$ can also be computed from the construction of $T_{0}$ given above:

$$
K_{T_{0}}=-4 H_{\mathrm{w}}+2 \hat{B}_{t, e}+\hat{C}_{t} .
$$

Thus we find:

$$
\hat{B}_{t, i}=12 H_{\mathrm{w}}-6 \hat{B}_{t, e}-3 \hat{C}_{t} .
$$

The $12 H_{\mathrm{w}}$ in this formula arises from the fact that each of the twelve divisors in $\hat{B}_{t, i}$ is the strict transform of plane in $\mathbf{P}_{\mathrm{w}}^{3}$. Since $\hat{B}_{t}=\hat{B}_{t, i}+2 \hat{B}_{t, e}$, this relation implies $4 \hat{B}_{t, e}=12 H_{\mathrm{w}}-\hat{B}_{t}-3 \hat{C}_{t}$ and thus we can eliminate $\hat{B}_{t, e}$ from the last formula for $K_{T_{0}}$ to obtain the formula in the theorem. The other Chern classes can be computed by restricting the Chern classes of $\mathcal{C}$ or directly by using the blow up formulas as in the proof of 5.3.

The restriction of the CAF-hyperplane class to $T_{0}$ is:

$$
\frac{1}{4}(\hat{B}+3 \hat{C})_{\mid T_{0}}=\frac{1}{4}\left(\hat{B}_{t, i}+2 \hat{B}_{t, e}+3 \hat{C}_{t}\right)=3 H_{\mathrm{w}}-\hat{B}_{t, e}
$$

which agrees with the fact that the CAF-map, when restricted to $\mathbf{P}_{\mathrm{w}}^{3}$, is given by the cubics which vanish on the 12 points which are blown up to give $\hat{B}_{t, e}([\mathrm{vG}]$, Thm 6.5).

6.5. Remark. The tritangent divisor $T_{0}$ also has non-empty intersection with the other $45-1=$ $12+2 \cdot 16$ tritangent divisors. It is verified in [vG], 6.7 that $2 \cdot 16$ tritangent divisors intersect $T_{0}$ in the strict transform of 16 quadrics in $\mathbf{P}_{\mathrm{w}}^{3}$.

The other 12 tritangent divisors intersect $\mathbf{P}_{\mathrm{w}}^{3}$ in the 12 linear subspaces defined by the short roots, that is by a pair $\pm \tau \in S$. The involutions corresponding to these 12 divisors are just the reflections in the corresponding short roots in $W\left(F_{4}\right) \subset W\left(E_{6}\right)$ ([vG], proof of Thm 6.5). The involution defined by $\tau$ permutes the boundary divisors $B_{ \pm \tau}$ (defined by $V( \pm \tau) \subset \tilde{T}$ ) in $\mathcal{C}$. Thus we conclude that the surface $V=T_{0} \cap B_{\tau} \cap B_{-\tau}$ must coincide with the divisor in $T_{0}$ defined by the plane in $\mathbf{P}_{\mathrm{w}}^{3}$ defined by the short root $\tau$. In particular, $V$ lies in two tritangent as well as in two boundary divisors.

The fact that there are surfaces in the intersection of pairs of boundary and tritangent divisors can also easily be seen using the ball quotient description of $\mathcal{C}$. Following [AF], the ball $\mathcal{B}$ is viewed as a subvariety of $\mathbf{C}^{5}$ :

$$
\mathcal{B}=\left\{\left(1, z_{1}, z_{2}, z_{3}, z_{4}\right) \in \mathbf{C}^{5}: 1-\left|z_{1}\right|^{2}-\ldots-\left|z_{4}\right|^{2}>0\right\}
$$

and we consider the hermitian form $(z, w)=z_{0} \bar{w}_{0}-z_{1} \bar{w}_{1}-\ldots-z_{4} \bar{w}_{4}$ on $\mathbf{C}^{5}$. Let $e_{1}=$ $(0,1,0,0,0), e_{2}=(0,0,1,0,0)$, then the subvarieties of the ball defined by $\left(z, e_{i}\right)=0$ map onto tritangent divisors $T_{i}$ under the map

$$
\mathcal{B} \longrightarrow \mathcal{B} / \Gamma \cong \mathcal{C}-\{\text { cusp divisors }\}
$$


The subvarieties defined by $w_{1}=e_{1}+e_{2}$ and $w_{2}=e_{1}-e_{2}$ map onto boundary divisors $T_{j}$. Obviously, the intersection of these four divisors is a surface.

\section{APPENDiX}

7.1. The divisor of $\lambda$. The divisor of the rational function $\lambda$, as well as $\lambda-1$, are used to obtain relations in the various Chow groups considered in this paper. In the toric variety $\tilde{T}$ we have

$$
(\lambda)=\sum_{\tau \in S \cup R} n_{\tau} V(\tau) \quad \text { with } \quad n_{\tau}=\left(\epsilon_{1}-\epsilon_{2}, \tau\right)
$$

(standard inner product on $\mathbf{R}^{4}$ ). Obviously, we have $n_{-\tau}=-n_{\tau}$, so it suffices to list the $\tau \in R \cap S$ with $n_{\tau}>0$ which is easily done.

$$
n_{\tau}>0 \quad \text { iff } \quad S_{\lambda, 0} \cup R_{\lambda, 0} \cup\left\{\epsilon_{1}-\epsilon_{2}\right\},
$$

where

$S_{\lambda, 0}=\left\{\epsilon_{1},-\epsilon_{2},\left(\epsilon_{1}-\epsilon_{2}+\epsilon_{3}+\epsilon_{4}\right) / 2,\left(\epsilon_{1}-\epsilon_{2}-\epsilon_{3}-\epsilon_{4}\right) / 2,\left(\epsilon_{1}-\epsilon_{2}+\epsilon_{3}-\epsilon_{4}\right) / 2,\left(\epsilon_{1}-\epsilon_{2}-\epsilon_{3}+\epsilon_{4}\right) / 2\right\}$,

here $\tau \in S$ so these give boundary divisors,

$$
R_{\lambda, 0}=\left\{\epsilon_{1}+\epsilon_{3}, \epsilon_{1}-\epsilon_{3}, \epsilon_{1}+\epsilon_{4}, \epsilon_{1}-\epsilon_{4},-\epsilon_{2}+\epsilon_{3},-\epsilon_{2}-\epsilon_{3},-\epsilon_{2}+\epsilon_{4},-\epsilon_{2}-\epsilon_{4}\right\}
$$

here $\tau \in R$ so these give cusp divisors, and finally $n_{\tau}=2$ if $\tau=\epsilon_{1}-\epsilon_{2}$, the divisor $V\left(\epsilon_{1}-\epsilon_{2}\right)$ is also a cusp divisor.

To find the divisor of the rational function $\lambda$ on $\mathcal{C}$, we observe that a divisor $V(\tau)$ does not intersect $T$, hence it does not contain $e \in T$ (which gets blown up in $\tilde{T}^{\prime}$ ) nor any of the 12 curves in $\tilde{T}^{\prime}$ which are blown up in $\tilde{T}^{\prime \prime}$ nor any of the 16 surfaces in $\tilde{T}^{\prime \prime}$ which are blown up in $\tilde{T}$. Thus the divisor of $\lambda$ in $\hat{T}$ is simply the sum of the strict transforms of the divisors $V(\tau) \subset \tilde{T}$ with the same multiplicities. Finally, the direct image under $r$ of this divisor in $\mathcal{C}$ is the divisor of $\lambda$ on $\mathcal{C}$.

Using the tables from 7.4 below one finds that

$S_{\lambda, 0}=\left\{h_{345}, h_{13}, h_{136}, h_{26}, h_{246}, h_{256}\right\} \subset\left\{h_{345}, h_{13}, h_{145}, h_{136}, h_{26}, h_{123}, h_{246}, h_{256}, h_{45}\right\}=[13.45 .26]$, that is, this set of 6 positive roots can be completed to a set three orthogonal $A_{2}$ 's (see 7.6). Similarly one finds

$S_{\lambda, \infty}=\left\{h_{12}, h_{245}, h_{36}, h_{126}, h_{356}, h_{346}\right\} \subset\left\{h_{12}, h_{245}, h_{145}, h_{36}, h_{126}, h_{123}, h_{356}, h_{346}, h_{45}\right\}=[12.45 .36]$, so, using the same three roots $h_{145}, h_{123}, h_{45}$, also this set can be completed to a set of three orthogonal $A_{2}$ 's. These sets of nine roots correspond to the cusp divisors $D_{v}$ where $v=$ $(1,-1,1,0,0) \in N_{0}\left(\right.$ with $\left.D_{v}=V\left(\epsilon_{1}-\epsilon_{2}\right)\right)$ and $w=(1,1,-1,0,0)\left(\right.$ with $\left.D_{w}=V\left(-\epsilon_{1}+\epsilon_{2}\right)\right)$ respectively. For $z \in N_{0}$ we define $\{z\}$ to be the set of nine (positive) roots in the corresponding $A_{2}^{3}$ and we put

$$
B_{z}=\sum_{t \in\{z\}} D_{t}
$$

Next we consider the cusp divisors. Using the table 7.6 we find that the eight $\tau \in$ $R_{\lambda, 0}$ correspond to the lines generated by the eight vectors $(1,-1,0, \pm 1,0),(1,-1,0,0, \pm 1)$, $(1,0,1, \pm 1,0),(1,0,1,0, \pm 1)$. These vectors are all perpendicular to $w$, similarly the $\tau \in R_{\lambda, \infty}$ correspond to the vectors $(1,1,0, \pm 1,0),(1,1,0,0, \pm 1),(1,0,-1, \pm 1,0),(1,0,-1,0, \pm 1)$ which are all in $v^{\perp}$. The linear space $v^{\perp} \cong \mathbf{F}_{3}^{4}$ contains 13 isotropic lines, 8 of these are spanned by 
the vectors listed, another 4 are spanned by $(0,1,1, \pm 1,0)$ and $(0,1,1,0, \pm 1)$ (note that these vectors are also in $w^{\perp}$ ) and finally there is the line spanned by $v$ itself. We write

$$
C_{v^{\perp}}=\sum_{z \in v^{\perp}} D_{z}
$$

for the sum of these 13 cusp divisors. Putting all this together, we have $(\lambda)=B_{v}+C_{w^{\perp}}+$ $3 D_{v}-\left(B_{w}+C_{v^{\perp}}+3 D_{w}\right)$.

As a byproduct of our labors we get:

$$
E_{v}=E_{w}, \quad\left(\in A^{1}(\mathcal{C})\right) \quad \text { with } \quad E_{v}=B_{v}-C_{v^{\perp}}+3 D_{v} .
$$

The group $W\left(E_{6}\right)$ permutes the $v \in N_{0}$ and it easily follows that any two $E_{v}$ 's are linearly equivalent, hence $E_{v}$ is an invariant class. Symmetrizing $E_{v}$ gives:

$$
E_{v}=\frac{9}{36} \hat{B}+\frac{(-13+3)}{40} \hat{C}=(\hat{B}-\hat{C}) / 4=-K_{\mathcal{C}} .
$$

7.2. The divisor of $\lambda-1$. The rational function $\lambda-1$ on has the same poles as $\lambda$. Its divisor of zeroes $(\lambda-1)$ in $\tilde{T}$ is the closure in $\tilde{T}$ of the subtorus defined by $\lambda=1$ in $T$, we denote it by $D_{\lambda}^{1}$. Since $\lambda(e)=1$, the divisor $(\lambda-1)_{0}$ in $\tilde{T}^{\prime}$, the blow up $\tilde{T}$ of $e$, is given by the sum of the strict transform of $D_{\lambda}^{1}$ and the exceptional fibre $\mathbf{P}_{\mathrm{w}}^{3}$. The strict transforms in $\hat{T}$ of the exceptional divisors for the map $\pi^{\prime}: \tilde{T}^{\prime \prime} \rightarrow \tilde{T}^{\prime}$ are blown down by $r: \tilde{T} \rightarrow \mathcal{C}$, hence in the end they do not contribute to $(\lambda-1)_{0}$ in $A^{1}(\mathcal{C})$. The map $\pi^{\prime \prime}$ blows up 16 surfaces in $\tilde{T}^{\prime}$, these are the strict transforms of surfaces in $\tilde{T}$. By inspection one finds that 4 of these are contained in $D_{\lambda}^{1}$, to be explicit, the defining equations of these curves are:

$$
\lambda=\mu \nu \rho=1, \quad \lambda=\mu \rho=1, \quad \lambda=\nu \rho=1, \quad \lambda=\rho=1 .
$$

Therefore the divisor $(\lambda-1)_{0}$ in $\hat{T}$ has 6 components (with multiplicity one), the strict transforms of $D_{\lambda}^{1}(\subset \tilde{T})$, of $\left.\mathbf{P}_{\mathrm{w}}^{3}\right)\left(\subset \tilde{T}^{\prime}\right)$ and the exceptional divisors over the 4 surfaces. The divisor $(\lambda-1)_{0}$ in $\mathcal{C}$ is the direct image of this divisor, in particular it is the sum of one boundary divisor (coming from $D_{\lambda}^{1}$ ), one tritangent divisor (coming from $\mathbf{P}_{\mathrm{w}}^{3}$ ) and 4 cusp divisors (coming from the exceptional divisors over the surfaces).

7.3. Tables. We collect some of the notations used by various authors for referring to tritangents, boundary and cusp divisors (cf. [Fr2] and $[\mathrm{S}]$ ). We also give a $W\left(E_{6}\right)$-equivariant map between the sets of these divisors and the sets $N_{i}$ (see 1.7).

7.4. The boundary divisors. The 36 boundary divisors are parametrized by the 36 positive roots of $E_{6}$. These roots generate the root lattice $Q\left(E_{6}\right) \cong \mathbf{Z}^{6}$. The quadratic form given by the Cartan matrix on $Q\left(E_{6}\right)$ has determinant 3, hence the $\mathbf{F}_{3}$-valued bilinear form it induces on $F F_{3}^{6}$ is degenerate. In fact, $v=\alpha_{1}-\alpha_{3}+\alpha_{5}-\alpha_{6}=h_{12}-h_{23}+h_{45}-h_{56} \in Q(R)$ has the property that $(v, \alpha) \in 3 \mathbf{Z}$ (this is easy to verify on a basis of simple roots of $E_{6}$ using the Dynkin diagram), but $v \notin 3 Q(R)$. More intrinsically, we note that $v / 3$ lies in the weight lattice $P\left(E_{6}\right)$ and that $P\left(E_{6}\right)=Q\left(E_{6}\right)+\mathbf{Z}(v / 3)$, hence $Q\left(E_{6}\right) /\left(3 Q\left(E_{6}\right)+\mathbf{Z} v\right) \cong Q\left(E_{6}\right) / 3 P\left(E_{6}\right)$ (cf. [B], Exercises, VI $\S 4$, n. 2). On $\mathbf{F}_{3}^{5} \cong Q\left(E_{6}\right) /\left(3 P\left(E_{6}\right)\right.$ we get a non-degenerate $\mathbf{F}_{3}$-valued quadratic form $q$ and we have an induced homomorphism $W\left(E_{6}\right) \rightarrow O\left(\mathbf{F}_{3}^{5}, q\right)$.

Under the quotient map

$$
\pi: Q(R) \longrightarrow Q\left(E_{6}\right) /\left(3 Q\left(E_{6}\right)+\mathbf{Z} v\right) \cong \mathbf{F}_{3}^{5}
$$


the roots of $E_{6}$ map to elements of $N_{-1}$. We fix $\pi$ by defining the images of the basis of simple roots $h_{12}, h_{123}, h_{23}, h_{34}, h_{45}, h_{56}$ :

$$
\pi\left(h_{i, i+1}\right)=f_{i}-f_{i+1} \quad(1 \leq i \leq 4), \quad \pi\left(h_{123}\right)=(0,0,0,1,1),
$$

here $f_{i}$ is the $i$-th standard basis vector of $\mathbf{F}_{3}^{5}$, and in view of $\pi(v)=0$ we get

$$
\pi\left(h_{56}\right)=h_{12}-h_{23}+h_{45}=(1,1,1,1,-1) .
$$

The first table lists the roots of $D_{4} \subset E_{6}$, the corresponding characters of $T$, the name of the root in $E_{6}$ and its image in $\mathbf{P}\left(\mathbf{F}_{3}^{5}\right)$. The last three tables list the elements in $S$, which define boundary divisors in $\mathcal{C}$, the corresponding positive root in $E_{6}$ and its image in $\mathbf{P}\left(\mathbf{F}_{3}^{5}\right)$. Note

\begin{tabular}{|c|c|c|c|c|c|c|}
\hline $\mathrm{e}_{3}+\mathrm{e}_{4}$ & $\mu$ & $h_{123}$ & $(0,0,0,1,1)$ & & & \\
\hline $\mathrm{e}_{3}-\mathrm{e}_{4}$ & $\nu$ & $h_{45}$ & $(0,0,0,1,-1)$ & & & \\
\hline $\mathrm{e}_{2}+\mathrm{e}_{4}$ & $\mu \rho$ & $h_{124}$ & $(0,0,1,0,1)$ & $\epsilon_{4}$ & $h_{234}$ & $(1,0,0,0,1)$ \\
\hline $\mathrm{e}_{2}-\mathrm{e}_{4}$ & $\nu \rho$ & $h_{35}$ & $(0,0,1,0,-1)$ & $-\epsilon_{4}$ & $h_{15}$ & $(1,0,0,0,-1)$ \\
\hline $\mathrm{e}_{2}+\mathrm{e}_{3}$ & $\mu \nu \rho$ & $h_{125}$ & $(0,0,1,1,0)$ & $\epsilon_{3}$ & $h_{235}$ & $(1,0,0,1,0)$ \\
\hline $\mathrm{e}_{2}-\mathrm{e}_{3}$ & $\rho$ & $h_{34}$ & $(0,0,1,-1,0)$ & $-\epsilon_{3}$ & $h_{14}$ & $(1,0,0,-1,0)$ \\
\hline $\mathrm{e}_{1}+\mathrm{e}_{4}$ & $\lambda \mu \rho$ & $h_{134}$ & $(0,1,0,0,1)$ & $\epsilon_{2}$ & $h_{245}$ & $(1,0,1,0,0)$ \\
\hline $\mathrm{e}_{1}-\mathrm{e}_{4}$ & $\lambda \nu \rho$ & $h_{25}$ & $(0,1,0,0,-1)$ & $-\epsilon_{2}$ & $h_{13}$ & $(1,0,-1,0,0)$ \\
\hline $\mathrm{e}_{1}+\mathrm{e}_{3}$ & $\lambda \mu \nu \rho$ & $h_{135}$ & $(0,1,0,1,0)$ & $\epsilon_{1}$ & $h_{345}$ & $(1,1,0,0,0)$ \\
\hline $\mathrm{e}_{1}-\mathrm{e}_{3}$ & $\lambda \rho$ & $h_{24}$ & $(0,1,0,-1,0)$ & $-\epsilon_{1}$ & $h_{12}$ & $(1,-1,0,0,0)$ \\
\hline $\mathrm{e}_{1}+\mathrm{e}_{2}$ & $\lambda \mu \nu \rho^{2}$ & $h_{145}$ & $(0,1,1,0,0)$ & & & \\
\hline $\mathrm{e}_{1}-\mathrm{e}_{2}$ & $\lambda$ & $h_{23}$ & $(0,1,-1,0,0)$ & & & \\
\hline
\end{tabular}
that $( \pm \pm \pm \pm)$ stands for the vector $\left( \pm \epsilon_{1} \pm \epsilon_{2} \pm \epsilon_{3} \pm \epsilon_{4}\right) / 2$.

\begin{tabular}{|c|c|c|c|c|c|}
\hline$(----)$ & $h$ & $(1,1,1,1,1)$ & $(+---)$ & $h_{26}$ & $(1,-1,1,1,1)$ \\
\hline$(---+)$ & $h_{56}$ & $(1,1,1,1,-1)$ & $(+--+)$ & $h_{256}$ & $(1,-1,1,1,-1)$ \\
\hline$(--+-)$ & $h_{46}$ & $(1,1,1,-1,1)$ & $(+-+-)$ & $h_{246}$ & $(1,-1,1,-1,1)$ \\
\hline$(--++)$ & $h_{456}$ & $(1,1,1,-1,-1)$ & $(+-++)$ & $h_{136}$ & $(1,-1,1,-1,-1)$ \\
\hline$(-+--)$ & $h_{36}$ & $(1,1,-1,1,1)$ & $(++--)$ & $h_{236}$ & $(1,-1,-1,1,1)$ \\
\hline$(-+-+)$ & $h_{356}$ & $(1,1,-1,1,-1)$ & $(++-+)$ & $h_{146}$ & $(1,-1,-1,1,-1)$ \\
\hline$(-++-)$ & $h_{346}$ & $(1,1,-1,-1,1)$ & $(+++-)$ & $h_{156}$ & $(1,-1,-1,-1,1)$ \\
\hline$(-+++)$ & $h_{126}$ & $(1,1,-1,-1,-1)$ & $(++++)$ & $h_{16}$ & $(1,-1,-1,-1,-1)$ \\
\hline
\end{tabular}

7.5. Tritangents. The tritangents were labelled by Cayley, later a more comprehensible notation was introduced by Schläfli. The 27 lines on a smooth cubic surface are denoted by $a_{1}, \ldots, a_{6}$ (these are 6 skew lines which can thus be blown down to points $\left.p_{i}\right), b_{1}, \ldots, b_{6}\left(b_{i}\right.$ maps to the conic on the 6 points except for $\left.p_{i}\right)$ and $c_{i j}, 1 \leq i<j \leq 6\left(c_{i j}\right.$ maps to the line $\left.\left\langle p_{i}, p_{j}\right\rangle\right)$. The 45 tritangent planes are denoted by

$$
(i j)=\left\langle a_{i}, b_{j}, c_{i j}\right\rangle, \quad(i j . k l . m n)=\left\langle c_{i j}, c_{k l}, c_{m n}\right\rangle,
$$

here $i \neq j$ and $(i j) \neq(j i)$, also $\{i, \ldots, n\}=\{1, \ldots, 6\}$ and $i<j, k<l, m<n$. A correspondence between Cayley's labels and those of Schläfli was given in [S] and is copied here.

Each tritangent plane is determined by a $D_{4} \subset E_{6}$ (cf. [vG], 1.8). The span of the image of such a $D_{4}$ in $\mathbf{F}_{3}^{5}$ has codimension one (the image of the 'standard' $D_{4}$ spanned by 
$h_{123}, h_{23}, h_{34}, h_{45}$ has this property, the other $D_{4}$ 's are in $W\left(E_{6}\right)$-orbit of this one), hence it is the perpendicular of a one dimensional subspace. In this way each tritangent defines a point in $\mathbf{P}\left(\mathbf{F}_{3}^{5}\right)$, one checks that this point lies in $N_{1}$ for the standard $D_{4}$, and thus it lies in $N_{1}$ for all tritangents. Alternatively, one can use $[\mathrm{AF}]$ to find this correspondence.

Another way to describe this correspondence was found by Matsumoto and Terasoma [MT], Prop. 3.4. They identify $\mathbf{F}_{3}^{5}$ with a subgroup of the 3-torsion points of the intermediate Jacobian of a cubic threefold associated to a cubic surface.

\begin{tabular}{|rrr|}
\hline$\xi$ & $(56)$ & $(0,0,0,0,1)$ \\
\hline$x$ & $(46)$ & $(0,0,0,1,0)$ \\
\hline $\mathrm{x}$ & $(36)$ & $(0,0,1,0,0)$ \\
\hline$\overline{\mathrm{x}}$ & $(26)$ & $(0,1,0,0,0)$ \\
\hline $\mathrm{w}$ & $(16)$ & $(1,0,0,0,0)$ \\
\hline$\overline{\mathrm{y}}$ & $(61)$ & $(0,1,1,1,1)$ \\
\hline$\overline{\mathrm{z}}$ & $(15)$ & $(0,1,1,1,-1)$ \\
\hline $\mathrm{z}$ & $(14)$ & $(0,1,1,-1,1)$ \\
\hline $\mathrm{y}$ & $(16.23 .45)$ & $(0,1,1,-1,-1)$ \\
\hline$z$ & $(13)$ & $(0,1,-1,1,1)$ \\
\hline$y$ & $(16.24 .35)$ & $(0,1,-1,1,-1)$ \\
\hline$\eta$ & $(16.25 .34)$ & $(0,1,-1,-1,1)$ \\
\hline$\zeta$ & $(12)$ & $(0,1,-1,-1,-1)$ \\
\hline$r_{1}$ & $(62)$ & $(1,0,1,1,1)$ \\
\hline$\overline{\mathrm{q}}_{1}$ & $(25)$ & $(1,0,1,1,-1)$ \\
\hline$\overline{\mathrm{m}}_{1}$ & $(24)$ & $(1,0,1,-1,1)$ \\
\hline $\mathrm{n}_{1}$ & $(13.26 .45)$ & $(1,0,1,-1,-1)$ \\
\hline$\overline{\mathrm{n}}_{\mathrm{n}}$ & $(23)$ & $(1,0,-1,1,1)$ \\
\hline$m$ & $(14.26 .35)$ & $(1,0,-1,1,-1)$ \\
\hline$\overline{\mathrm{q}}$ & $(15.26 .34)$ & $(1,0,-1,-1,1)$ \\
\hline$\overline{\mathrm{r}}$ & $(21)$ & $(1,0,-1,-1,-1)$ \\
\hline & &
\end{tabular}

\begin{tabular}{|rrr|}
\hline$\overline{\mathrm{n}}_{1}$ & $(63)$ & $(1,1,0,1,1)$ \\
\hline $\mathrm{m}_{1}$ & $(35)$ & $(1,1,0,1,-1)$ \\
\hline $\mathrm{q}_{1}$ & $(34)$ & $(1,1,0,-1,1)$ \\
\hline $\mathrm{r}_{1}$ & $(12.36 .45)$ & $(1,1,0,-1,-1)$ \\
\hline $\mathrm{r}$ & $(32)$ & $(1,-1,0,1,1)$ \\
\hline $\mathrm{q}$ & $(14.25 .36)$ & $(1,-1,0,1,-1)$ \\
\hline$\overline{\mathrm{m}}$ & $(15.24 .36)$ & $(1,-1,0,-1,1)$ \\
\hline $\mathrm{n}$ & $(31)$ & $(1,-1,0,-1,-1)$ \\
\hline$\overline{\mathrm{l}}$ & $(64)$ & $(1,1,1,0,1)$ \\
\hline $\mathrm{l}$ & $(45)$ & $(1,1,1,0,-1)$ \\
\hline$\overline{\mathrm{g}}$ & $(43)$ & $(1,1,-1,0,1)$ \\
\hline$\overline{\mathrm{h}}$ & $(12.35 .46)$ & $(1,1,-1,0,-1)$ \\
\hline $\mathrm{h}$ & $(42)$ & $(1,-1,1,0,1)$ \\
\hline $\mathrm{g}$ & $(13.25 .46)$ & $(1,-1,1,0,-1)$ \\
\hline$\overline{\mathrm{l}}_{1}$ & $(15.23 .46)$ & $(1,-1,-1,0,1)$ \\
\hline $\mathrm{l}_{1}$ & $(41)$ & $(1,-1,-1,0,-1)$ \\
\hline$\overline{\mathrm{p}}$ & $(65)$ & $(1,1,1,1,0)$ \\
\hline $\mathrm{p}$ & $(54)$ & $(1,1,1,-1,0)$ \\
\hline $\mathrm{f}$ & $(53)$ & $(1,1,-1,1,0)$ \\
\hline$\theta$ & $(12.34 .56)$ & $(1,1,-1,-1,0)$ \\
\hline $\bar{\theta}$ & $(52)$ & $(1,-1,1,1,0)$ \\
\hline$\overline{\mathrm{f}}$ & $(13.24 .56)$ & $(1,-1,1,-1,0)$ \\
\hline $\mathrm{p} 1$ & $(14.23 .56)$ & $(1,-1,-1,1,0)$ \\
\hline$\overline{\mathrm{p}} 1$ & $(51)$ & $(1,-1,-1,-1,0)$ \\
\hline & & \\
\hline
\end{tabular}

7.6. The cusp divisors. The 40 cusp divisors can be labelled by 'triads of trihedral pairs' (cf. $[\mathrm{H}], 6.1 .1$ ), they correspond also to copies of three orthogonal $A_{2}$ 's in $E_{6}$ (cf. $\left.[\mathrm{H}], 6.1 .5 .3\right)$ The notation we use is:

$$
[i j k . l m n]=\left[\begin{array}{ccc}
h_{i j} & h_{j k} & h_{i k} \\
h_{l m} & h_{m n} & h_{l n} \\
h & h_{i j k} & h_{l m n}
\end{array}\right], \quad[i j . k l . m n]=\left[\begin{array}{ccc}
h_{i j} & h_{i k l} & h_{j k l} \\
h_{k l} & h_{k m n} & h_{l m n} \\
h_{m n} & h_{n i j} & h_{m i j}
\end{array}\right],
$$

here the rows of the matrices are thee positive vectors of $E_{6}$ which span an $A_{2}$ and the roots in different rows are perpendicular.

The image under $\pi$ of the span of an $A_{2} \subset Q\left(E_{6}\right)$ is a two dimensional subspace of $\mathbf{F}_{3}^{5}$, its projectivizations thus has 4 points, three of which are lines spanned by the positive roots, the other line is spanned by an element from $N_{0}$. The span of the images of three perpendicular 
$A_{2}$ 's has codimension one in $\mathbf{F}_{3}^{5}$ (in fact, the span of each of the three contains the same element $v$ from $N_{0}$ ). Hence this subspace is the perpendicular of an element, which in fact is this $v \in N_{0}$ (to check this it suffices to verify this statement for one of the 40 triples, and to apply $W\left(E_{6}\right)$ to obtain the result in general). Conversely, given a vector $v \in N_{0}$, it is perpendicular to exactly 9 elements in $N_{-1}$.

The first table lists the equations of a subtorus of $T$ whose closure in the toric variety $\tilde{T}$ is one of the 16 surfaces $S_{i}$, the pair of roots in $D_{4} \subset E_{6}$ which define the surface are also given. This pair of roots spans an $A_{2} \subset E_{6}$ which is contained in a unique triple of orthogonal $A_{2}$ 's. The corresponding cusp divisor in $\mathcal{C}$ is then labelled as above as well as by a vector in $N_{0}$, this vector is normalized by its first non-zero component being +1 . The last table lists a $\tau \in R$, which defines a cusp divisor in $\mathcal{C}$, a label for the corresponding triple of orthogonal $A_{2}$ 's as well as the corresponding normalized vector in $N_{0}$.

\begin{tabular}{|r|r|r|r|}
\hline$\nu=\rho=1$ & $h_{34}, h_{45}$ & {$[126.345]$} & $(0,0,1,1,1)$ \\
\hline$\mu=\rho=1$ & $h_{34}, h_{123}$ & {$[34.12 .56]$} & $(0,0,1,1,-1)$ \\
\hline$\mu=\nu \rho=1$ & $h_{35}, h_{123}$ & {$[35.12 .46]$} & $(0,0,1,-1,1)$ \\
\hline$\nu=\mu \rho=1$ & $h_{45}, h_{124}$ & {$[45.12 .36]$} & $(0,0,-1,1,1)$ \\
\hline$\nu=\lambda \rho=1$ & $h_{24}, h_{45}$ & {$[136.245]$} & $(0,1,0,1,1)$ \\
\hline$\mu=\lambda \rho=1$ & $h_{24}, h_{123}$ & {$[24.13 .56]$} & $(0,1,0,1,-1)$ \\
\hline$\mu=\lambda \nu \rho=1$ & $h_{123}, h_{25}$ & {$[25.13 .46]$} & $(0,1,0,-1,1)$ \\
\hline$\nu=\lambda \mu \rho=1$ & $h_{45}, h_{134}$ & {$[45.13 .26]$} & $(0,1,0,-1,-1)$ \\
\hline$\lambda=\nu \rho=1$ & $h_{23}, h_{35}$ & {$[146.235]$} & $(0,1,1,0,1)$ \\
\hline$\lambda=\mu \rho=1$ & $h_{23}, h_{124}$ & {$[23.14 .56]$} & $(0,1,1,0,-1)$ \\
\hline$\lambda \nu \rho=\mu \rho=1$ & $h_{25}, h_{124}$ & {$[25.14 .56]$} & $(0,1,-1,0,1)$ \\
\hline$\lambda \mu \rho=\nu \rho=1$ & $h_{35}, h_{134}$ & {$[35.14 .56]$} & $(0,1,-1,0,-1)$ \\
\hline$\lambda=\rho=1$ & $h_{23}, h_{34}$ & {$[234.156]$} & $(0,1,1,1,0)$ \\
\hline$\lambda=\mu \nu \rho=1$ & $h_{23}, h_{125}$ & {$[23.15 .46]$} & $(0,1,1,-1,0)$ \\
\hline$\mu \nu \rho=\lambda \rho=1$ & $h_{24}, h_{125}$ & {$[24.15 .36]$} & $(0,1,-1,1,0)$ \\
\hline$\rho=\lambda \mu \nu \rho=1$ & $h_{34}, h_{135}$ & {$[34.15 .26]$} & $(0,1,-1,-1,0)$ \\
\hline
\end{tabular}

\begin{tabular}{|rrr|}
\hline$-\epsilon_{3}-\epsilon_{4}$ & {$[145.236]$} & $(1,0,0,1,1)$ \\
\hline$-\epsilon_{3}+\epsilon_{4}$ & {$[14.23 .56]$} & $(1,0,0,1,-1)$ \\
\hline$\epsilon_{3}-\epsilon_{4}$ & {$[15.23 .46]$} & $(1,0,0,-1,1)$ \\
\hline$\epsilon_{3}+\epsilon_{4}$ & {$[45.23 .16]$} & $(1,0,0,-1,-1)$ \\
\hline$-\epsilon_{2}-\epsilon_{4}$ & {$[135.246]$} & $(1,0,1,0,1)$ \\
\hline$-\epsilon_{2}+\epsilon_{4}$ & {$[13.24 .56]$} & $(1,0,1,0,-1)$ \\
\hline$\epsilon_{2}-\epsilon_{4}$ & {$[15.24 .36]$} & $(1,0,-1,0,1)$ \\
\hline$\epsilon_{2}+\epsilon_{4}$ & {$[35.24 .16]$} & $(1,0,-1,0,-1)$ \\
\hline$-\epsilon_{2}-\epsilon_{3}$ & {$[134.256]$} & $(1,0,1,1,0)$ \\
\hline$-\epsilon_{2}+\epsilon_{3}$ & {$[13.25 .46]$} & $(1,0,1,-1,0)$ \\
\hline$\epsilon_{2}-\epsilon_{3}$ & {$[14.25 .36]$} & $(1,0,-1,1,0)$ \\
\hline$\epsilon_{2}+\epsilon_{3}$ & {$[34.25 .16]$} & $(1,0,-1,-1,0)$ \\
\hline
\end{tabular}

\begin{tabular}{|rrr|}
\hline$-\epsilon_{1}-\epsilon_{4}$ & {$[125.346]$} & $(1,1,0,0,1)$ \\
\hline$-\epsilon_{1}+\epsilon_{4}$ & {$[12.34 .56]$} & $(1,1,0,0,-1)$ \\
\hline$\epsilon_{1}-\epsilon_{4}$ & {$[15.34 .26]$} & $(1,-1,0,0,1)$ \\
\hline$\epsilon_{1}+\epsilon_{4}$ & {$[25.34 .16]$} & $(1,-1,0,0,-1)$ \\
\hline$-\epsilon_{1}-\epsilon_{3}$ & {$[124.356]$} & $(1,1,0,1,0)$ \\
\hline$-\epsilon_{1}+\epsilon_{3}$ & {$[12.35 .46]$} & $(1,1,0,-1,0)$ \\
\hline$\epsilon_{1}-\epsilon_{3}$ & {$[14.35 .26]$} & $(1,-1,0,1,0)$ \\
\hline$\epsilon_{1}+\epsilon_{3}$ & {$[24.35 .16]$} & $(1,-1,0,-1,0)$ \\
\hline$-\epsilon_{1}-\epsilon_{2}$ & {$[123.456]$} & $(1,1,1,0,0)$ \\
\hline$-\epsilon_{1}+\epsilon_{2}$ & {$[12.45 .36]$} & $(1,1,-1,0,0)$ \\
\hline$\epsilon_{1}-\epsilon_{2}$ & {$[13.45 .26]$} & $(1,-1,1,0,0)$ \\
\hline$\epsilon_{1}+\epsilon_{2}$ & {$[23.45 .16]$} & $(1,-1,-1,0,0)$ \\
\hline
\end{tabular}




\section{REFERENCES}

[ACT] D. Allcock, J.A. Carlson, D. Toledo, The Complex Hyperbolic Geometry of the Moduli Space of Cubic Surfaces, eprint math.AG/0007048, to appear in: Journal of Algebraic Geometry.

[AF] D. Allcock and E. Freitag, Cubic surfaces and Borcherds products, eprint math.AG/0002066.

[At] J. H. Conway et al., Atlas of finite groups. Oxford University Press, 1985.

[B] N. Bourbaki, Groupes et Algèbras de Lie, Chapitres 4,5 et 6. Masson, Paris, (1981).

[F] J. S. Frame, The classes and representations of the groups of 27 lines and 28 bitangents. Ann. Mat. Pura Appl. 32, 83-119 (1951).

[Fr] E. Freitag, A graded algebra related to cubic surfaces, Kyushu J. Math. 56, 299-312 (2002).

[Fr2] E. Freitag, Comparison of different models of the moduli space of marked cubic surfaces, In:Proceedings of Japanese-German Seminar Explicit Structures of Modular Forms and Zeta Functions, Editors: T. Ibukiyama and W. Kohnen. Ryushi-do, 2002.

[Fu1] W. Fulton, Intersection theory. Springer-Verlag, Berlin-New York, (1984).

[Fu2] W. Fulton, Introduction to toric varieties. Annals of Mathematics Studies, 131. Princeton University Press, 1993.

[vG] B. van Geemen, A linear system on Naruki's moduli space of marked cubic surfaces. Internat. J. Math. 13, 183-208 (2002).

[GH] Ph. Griffiths, J. Harris, Principles of algebraic geometry. John Wiley \& Sons, New York, 1978.

$[\mathrm{H}] \quad$ B. Hunt, The geometry of some special arithmetic quotients. Lecture Notes in Mathematics, 1637. Springer-Verlag, Berlin, (1996).

[HAG] R. Hartshorne, Algebraic geometry. GTM 52, Springer-Verlag, New York-Heidelberg, 1977.

[L] E. Looijenga, Affine Artin groups and the fundamental groups of some moduli spaces, eprint math.AG/9801117.

[MT] K. Matsumoto, T. Terasoma, Theta constants associated to cubic three folds, eprint math.AG/0008024.

[N] I. Naruki, Cross ratio variety as a moduli space of cubic surfaces, Appendix by E. Looijenga, Proc. Lond. Math. Soc. 45, 1-30, (1982).

[S] J. Sekiguchi, The versal deformation of the $E_{6}$-singularity and a family of cubic surfaces. J. Math. Soc. Japan 46 (1994), 355-383.

Dipartimento di Matematica, Università di Milano, via Saldini 50, I-20133 Milano, Italia

E-mail address: Elisabetta.Colombo@mat.unimi.it

Dipartimento di Matematica, Università di Milano, via Saldini 50, I-20133 Milano, Italia

E-mail address: geemen@mat.unimi.it 